\title{
Dislocation multiplication by cross-slip and glissile reaction in a dislocation based continuum formulation of crystal plasticity
}

\author{
Markus Sudmanns ${ }^{\mathrm{a}}$, Markus Stricker ${ }^{\mathrm{c}}$, Daniel Weygand ${ }^{\mathrm{a}}$, Thomas Hochrainer ${ }^{\mathrm{b}}$, Katrin Schulz ${ }^{\mathrm{a}, *}$ \\ ${ }^{a}$ Institute for Applied Materials, Karlsruhe Institute of Technology, Kaiserstraße 12, 76131 Karlsruhe, Germany \\ ${ }^{b}$ Institut für Festigkeitslehre, Technische Universität Graz, Kopernikusgasse 24, 8010 Graz, Austria \\ ${ }^{c}$ Institute of Mechanical Engineering, École Polytechnique Fédérale de Lausanne, CH-1015, Switzerland
}

\begin{abstract}
Modeling dislocation multiplication due to interaction and reactions on a mesoscopic scale is an important task for the physically meaningful description of stage II hardening in face-centered cubic crystalline materials. In recent Discrete Dislocation Dynamics simulations it is observed that dislocation multiplication is exclusively the result of mechanisms, which involve dislocation reactions between different slip systems. These findings contradict multiplication models in dislocation based continuum theories, in which density increase is related to plastic slip on the same slip system. An application of these models for the density evolution on individual slip systems results in self-replication of dislocation density. We introduce a formulation of dislocation multiplication in a dislocation based continuum formulation of plasticity derived from a mechanism-based homogenization of cross-slip and glissile reactions in three-dimensional facecentered cubic systems. As a key feature, the presented model includes the generation of dislocations based on an interplay of dislocation density on different slip systems. This particularly includes slip systems with vanishing shear stress. The results show, that the proposed dislocation multiplication formulation allows for a physically meaningful microstructural evolution without self-replication of dislocations density. The results are discussed in comparison to discrete dislocation dynamics simulations exposing the coupling of different slip systems as the central characteristic for the increase of dislocation density on active and inactive slip systems.
\end{abstract}

Keywords:

Crystal plasticity, Continuum dislocation dynamics, Dislocation multiplication, Dislocation interaction

\section{Introduction}

One of the most salient features of metal-plasticity is the phenomenon of work-hardening. It is well known that work-hardening is the result of dislocation multiplication during plastic deformation. However, the complexity of dislocation interactions during work-hardening have 5 as yet precluded the development of a dislocation-based theory of work hardening in single crystals. Recent Discrete Dislocation Dynamics (DDD) simulations reveal details of dislocation

${ }^{*}$ Corresponding author: katrin.schulz@kit.edu 
multiplication in face-centered cubic (fcc) single crystals which question traditional pictures of and models for dislocation multiplication and work-hardening in crystal plasticity [1]. The most important findings of the named study are $(i)$ dislocation multiplication in the sense of emergence of "new" dislocation loops, as opposed to mere line-length increase, is exclusively due to cross5 slip and the formation of glissile junctions. (ii) Dislocations only sweep small areas within their slip system before getting involved in further reactions. These reactions seldomly lead to storage of immobilized dislocations but rather spawn dislocations on other slip systems.

The above named findings contrast assumptions of various continuum dislocation models used in crystal plasticity. In many dislocation-based crystal plasticity models $[2,3,4,5,6]$ 10 dislocation multiplication is modeled by a slip-system-wise adoption of the phenomenological Kocks-Mecking [7] theory. Because line length increase in these models is related to plastic slip, dislocation multiplication on any slip system is solely driven by slip on the same slip system. Densities on other slip systems only affect the density production related to plastic slip on a given slip system by contributing to what is called the "mean free path" of the slip system. No 15 transfer of density between slip systems may be considered. This is in contrast to the above named observation $(i)$. Note, moreover, that the Kocks-Mecking type models are rooted in the idea of dislocation storage after having traveled on average the length of the mean free path, which was likewise not corroborated in the named DDD simulations, cf. point (ii).

It may not be surprising that phenomenological models, as the one by Kocks and Mecking and its derivates, which are necessarily ignorant to microscopic details, do not comply with the latter. But also recent models which seek for a detailed representation of dislocation kinematics do not yet reflect the observations of [1]. Arsenlis and Parks [8] introduced a continuum formulation of plasticity, which is based on connected straight edge and screw dislocation segments. Thereby, the dislocation line length of one type is increased by the motion of the other disloca-

25 tion type, respectively. Based on [8], enhanced models have been proposed e.g. by [5, 9, 10] which, however, do not change the underlying idea of how line length is increased on the given slip system. Other aspects of dislocation multiplication have occasionally been incorporated in these edge-screw models. A model for cross-slip was introduced by [9] based on the kinematic formulation from Arsenlis and Parks [8]. The model is based on the assumption that the fraction

30 of screw dislocation density on the slip systems is always proportional to the respective probabilities of cross-slip. However, since the cross-slip process is not explicitly modeled as an actual transfer of density between respective slip systems, the dislocation density increase is still based on plastic slip on the same slip system.

In contrast to the above mentioned edge-screw approaches, the Continuum Dislocation Dynamics (CDD) theory $[11,12,13]$ is based on the kinematics of curved dislocation lines. It provides the foundation to formulate crystal plasticity based on moving closed dislocation lines in three dimensional systems. Besides the dislocation line length, CDD comprises the change in dislocation line orientation along the dislocation lines, as represented in an average sense by the so called curvature density. The curvature density contributes to the change in line length

40 during evolution (expansion or shrinkage) of closed dislocation loops. CDD has been shown to reproduce non-trivial details of dislocation density evolution in comparison to simplified DDD simulations [14]. Extensions by the incorporation of elastic dislocation interaction have been presented in $[15,16,17,18,13]$.

However, the curvature density in planar CDD is a conserved quantity; and for systems of 45 dislocation loops of a common line sense, the integral of the curvature density is proportional to the total number of loops on a slip system. CDD therefore conserves the number of dislocation loops. Multiplication in the sense of the generation of new dislocation loops is therefore not 
yet incorporated in the CDD evolution equations. A few attempts have been made to introduce new dislocations in the sense of new topologically closed objects to the CDD evolution equations. Early attempts were usually based on the idea of Frank-Read sources, e.g. [19, 20, 21]. However, the picture of a fixed source, which repeatedly produces new dislocations contradicts the obser5 vation of dislocation multiplication in a regime, which is characterized by complex dislocation networks and multiple active slip systems [1]. Note also, that no detachment of dislocations from their initial site (either glissile junction or cross-slip position) were observed in the recent DDD study. Recently, CDD-based formulations accounting for dislocation annihilation via climb and cross-slip as well as dislocation multiplication have been presented by [22]. However, the pro-

10 posed multiplication formulations are still based on the picture of Frank-Read sources, which has been shown to be inappropriate [1]. Furthermore the multiplication terms of [22] only affect the curvature density such that no transfer of line length (i.e. dislocation density) occurs by the reaction mechanisms themselves. A deposition of reaction products on inactive slip systems, as observed e.g. in [1], may therefore not be reproduced from these terms.

In this paper, we extend a model of dislocation multiplication [23] for use in CDD theory, which incorporates key observations from the DDD simulations [1]. The model is based on a homogenized description of glissile reactions and cross-slip and describes dislocation multiplication as generation of new dislocation density based on the dislocation density on the involved slip systems. The formulation is analyzed and compared to DDD simulations [1]. It is shown that the central feature of the model - the homogenized coupling of different slip systems to produce new dislocation density and curvature density - enables a physical description of dislocation multiplication. In contrast to existing models, the interplay of dislocation densities on different slip systems is represented, which enables the dislocation density increase on inactive slip systems to contribute to further dislocation interaction mechanisms.

\section{Method}

\subsection{Dislocation based continuum model}

We describe the elasto-plastic deformation behavior of face-centered cubic (fcc) metals, where plastic distortions solely results from the evolution of the dislocation state, as represented by the CDD density variables. The formulation is based on the additive decomposition of the

so small-strain distortion tensor into an elastic and a plastic part

$$
\mathrm{Du}=\boldsymbol{\beta}_{\mathrm{el}}+\boldsymbol{\beta}_{\mathrm{pl}}
$$

The plastic distortion tensor $\boldsymbol{\beta}_{\mathrm{pl}}$ is determined by evaluating the sum of plastic shear over all slip systems $\xi$

$$
\boldsymbol{\beta}_{\mathrm{pl}}=\sum_{\xi=1}^{N} \gamma^{\xi} \mathbf{d}^{\xi} \otimes \mathbf{m}^{\xi} .
$$

Herein, the orientations of the slip systems are defined according to the fcc microstructure by the orthonormal basis $\left\{\mathbf{d}^{\xi}, \mathbf{l}^{\xi}, \mathbf{m}^{\xi}\right\}$ with the slip plane normal $\mathbf{m}^{\xi}$ and the slip direction $\mathbf{d}^{\xi}=\frac{1}{b} \mathbf{b}^{\xi}$,

35 where $\mathbf{b}^{\xi}$ is the Burgers vector of length $b=\left|\mathbf{b}^{\xi}\right|$ and $\mathbf{l}^{\xi}=\mathbf{m}^{\xi} \times \mathbf{d}^{\xi}$. The plastic shear $\gamma^{\xi}$ is the result of dislocation motion on the respective slip systems $\xi$ and thus couples dislocation motion with plastic deformation.

The dislocation microstructure is characterized on each slip system $\xi$ by the total dislocation density $\rho^{\xi}$, the vector of the geometrically necessary dislocation density $\boldsymbol{\kappa}^{\xi}=\left(\kappa_{\text {screw }}^{\xi}, \kappa_{\text {edge }}^{\xi}, 0\right)$, and 
the curvature density $q^{\xi}$, which describes the local change in angular orientation of an ensemble of dislocation lines in an averaging volume. The evolution of the averaged dislocation state is given by the flux-based evolution equations in conservative form for $q^{\xi}$ [12], based on the isotropic dislocation velocity $v^{\xi}$,

$$
\begin{aligned}
& \partial_{t} \rho^{\xi}=-\nabla \cdot\left(v^{\xi} \boldsymbol{\kappa}_{\perp}^{\xi}\right)+v^{\xi} q^{\xi} \quad \text { with } \quad \boldsymbol{\kappa}_{\perp}^{\xi}=\boldsymbol{\kappa}^{\xi} \times \mathbf{m}^{\xi} \\
& \partial_{t} \boldsymbol{\kappa}^{\xi}=\nabla \times\left(\rho^{\xi} v^{\xi} \mathbf{m}^{\xi}\right) \\
& \partial_{t} q^{\xi}=-\nabla \cdot\left(\frac{q^{\xi}}{\rho^{\xi}} \boldsymbol{\kappa}_{\perp}^{\xi} v^{\xi}+\boldsymbol{A}^{\xi} \nabla v^{\xi}\right) .
\end{aligned}
$$

5 In the evolution of $q^{\xi}$ we employ the closure assumptions introduced in [11], with the dislocation alignment tensor

$$
\boldsymbol{A}^{\xi}=\frac{1}{2\left|\boldsymbol{\kappa}^{\xi}\right|^{2}}\left(\left(\rho^{\xi}+\left|\boldsymbol{\kappa}^{\xi}\right|\right) \boldsymbol{\kappa}^{\xi} \otimes \boldsymbol{\kappa}^{\xi}+\left(\rho^{\xi}-\left|\boldsymbol{\kappa}^{\xi}\right|\right) \boldsymbol{\kappa}_{\perp}^{\xi} \otimes \boldsymbol{\kappa}_{\perp}^{\xi}\right)
$$

The evolution of plastic shear $\gamma^{\xi}$, is obtained from Orowan's equation,

$$
\partial_{t} \gamma^{\xi}=v^{\xi} b \rho^{\xi}
$$

The evolution equations are closed by the formulation of a velocity law, which characterizes the relationship between the stresses and the velocity of the dislocation density. Here, we assume a linear dependency between the effective resolved shear stress $\tau^{\xi}$ and the velocity $v^{\xi}$ on each slip system, when the effective resolved shear stress exceeds the yield stress (see next paragraph) on the slip system. The effective resolved shear stress represents the superposition of a shear stress due to external loading and internal shear stresses of the dislocation microstructure, $\tau^{\xi}=\tau_{\mathrm{ext}}^{\xi}+\tau_{\mathrm{int}}^{\xi}$. The internal stress field $\tau_{\mathrm{int}}^{\xi}$, is divided into long-range and short-range 5 stresses in the continuum model. Long range stresses are represented using a mean field approach as in [16], which is used to resolve the mean dislocation stress field according to the numerical resolution of the system. Thus, the approach introduces a mesh dependent stress component and therefore loses accuracy during coarse graining. For systems with strong gradients of geometrically necessary dislocations (GNDs), this can be enhanced by short-range correction

20 stresses [16]. Furthermore, the long-range stress field vanishes in configurations, which only consist of statistically stored dislocations (SSDs).

To incorporate the hardening due to dislocation interaction between different slip systems, we use an additional stress term $\tau_{\mathrm{y}}$, which reduces the dislocation velocity due to the interaction with forest dislocations. This stress can be interpreted as a local yield stress and is incorporated 25 into the velocity law as

$$
v^{\xi}= \begin{cases}\frac{b}{B}\left(\left|\tau^{\xi}\right|-\tau_{\mathrm{y}}\right) \operatorname{sign}\left(\tau^{\xi}\right) & \text { if }\left|\tau^{\xi}\right|>\tau_{\mathrm{y}} \\ 0 & \text { if }\left|\tau^{\xi}\right| \leq \tau_{\mathrm{y}}\end{cases}
$$

Following [18], the yield stress $\tau_{y}=G b \alpha \sqrt{\rho}$ is applied based on [7, 24] for the system considered in this paper with the shear modulus $G$ and the constant $\alpha=0.35$, since it has been shown that the "Taylor" averaging of the forest interaction is a reasonable simplification for high-symmetry systems. 
The evolution equations, which describe the microstructural evolution, are incorporated into the numerical framework described in [25] using the finite element code $\mathrm{M}++$ based on a parallel multigrid method [26, 27]. The evolution equations of the dislocation densities Eq. (3) are combined into a system of equations

$$
\partial_{t}\left(\begin{array}{c}
\rho^{\xi} \\
\boldsymbol{\kappa}^{\xi}
\end{array}\right)+\nabla \cdot \boldsymbol{F}^{\xi}\left(\begin{array}{c}
\rho^{\xi} \\
\boldsymbol{\kappa}^{\xi}
\end{array}\right)=\boldsymbol{G}^{\xi}
$$

5 with the flux $\boldsymbol{F}^{\xi}$ and the source $\boldsymbol{G}^{\xi}$ given as

$$
\boldsymbol{F}^{\xi}\left(\begin{array}{c}
\rho^{\xi} \\
\boldsymbol{\kappa}^{\xi}
\end{array}\right)=v^{\xi}\left(\begin{array}{c}
\mathbf{l}^{\xi} \cdot \boldsymbol{\kappa}^{\xi} \mathbf{d}^{\xi}-\mathbf{d}^{\xi} \cdot \boldsymbol{\kappa}^{\xi} \mathbf{l}^{\xi} \\
\left(\mathbf{d}^{\xi} \otimes \mathbf{l}^{\xi}-\mathbf{l}^{\xi} \otimes \mathbf{d}^{\xi}\right) \rho^{\xi}
\end{array}\right) \text { and } \boldsymbol{G}^{\xi}=\left(\begin{array}{c}
v^{\xi} q^{\xi} \\
0
\end{array}\right) \text {. }
$$

Herein, the right hand side of Eq. (7) is a production term depending on the curvature density $q^{\xi}$, whose evolution we treat in a separate transport problem. For a given velocity $v^{\xi}$ and alignment tensor $\boldsymbol{A}^{\xi}$, we then obtain the transport equation of the curvature density

$$
\partial_{t} q^{\xi}+\nabla \cdot \boldsymbol{f}^{\xi}\left(q^{\xi}\right)=g^{\xi}
$$

with the flux term $\boldsymbol{f}^{\xi}\left(q^{\xi}\right)=\frac{q^{\xi}}{\rho^{\xi}} \boldsymbol{\kappa}_{\perp}^{\xi} v^{\xi}$ and the rotation term $g^{\xi}=-\nabla \cdot \boldsymbol{A}^{\xi} \nabla v^{\xi}$. Using a discontinuous 10 Galerkin scheme, the equations are solved separately on each slip system. Stresses are computed by a standard finite element solver, where the same mesh is used for the stress computation and the evaluation of the internal variables of the microstructure.

\subsection{Homogenized model of dislocation multiplication mechanisms}

For a better understanding, it is necessary to introduce a clear definition of the terms and their interpretation as continuum variables used in the following. In analogy to [1], we henceforth reserve the term dislocation for the boundary of a slipped area within a slip plane and is always a closed loop. The interpretation of dislocations as closed objects is distinguished from the dislocation line, which denotes the directed defect line of which the dislocation consists. Using these definitions, the model of dislocation multiplication in this paper is based on an analysis of

20 DDD simulations [1]. The main observations from this study include: (1) The key mechanisms, which provides new dislocations are cross-slip and glissile junctions, which both involve an interplay between different slip systems. (2) The generated dislocations show a limited expansion before most of their line length is annihilated or involved in further multiplication events. (3) The remaining dislocation lines are mostly straight segments in a stationary dislocation network, in

25 which a large part of the angular change, i.e. curvature along the dislocation line, is contained in the end-nodes of dislocation junctions. (4) Continued plasticity needs a continued generation of new dislocations.

The terminological distinction between dislocation lines and dislocations is captured by two different continuum variables in the CDD theory, namely the dislocation density $\rho^{\xi}$ (describing dislocation line length) and the curvature density $q^{\xi}$. The curvature density describes the local change in angular orientation of the ensembles of dislocation lines and the integral of $q^{\xi}$ over the simulation volume can be interpreted as the total angular content in the system. If all dislocation lines have the same line sense, this integral value divided by $2 \pi$ can be interpreted as the number of closed dislocation loops in the system. Currently, the kinematics of the CDD formulation is

35 based on the interconnection of all line orientations of a dislocation loop on the same slip system 
by the curvature density $q^{\xi}$. Hence, the evolution of the dislocation densities is restricted to the respective slip systems.

As stated in [1], the observations in the DDD study have consequences for the development of a multiplication model in dislocation based continuum theories and the kinematics of a con-

tinuum theory itself. In contrast to the current CDD kinematics, most of the contribution to the angular content of the dislocations in DDD is concentrated at junction nodes, which are equilibrated within the three dimensional dislocation network [1]. This includes the glissile and crossslip reactions. A dislocation in DDD can consist of physical and virtual line length. The physical line length denotes the length of the observable dislocation line, whereas the virtual line length 10 limits the slipped area of a dislocation along dislocation junctions or cross-slipped dislocation lines and therefore serves as a coupling element between the slip systems [1]. In contrast, the current CDD formulation does not incorporate quantities, which could be interpreted as 'virtual', i.e. all quantities are 'physical'. Therefore, the CDD kinematics based on dislocations, which are closed on the same slip system are in conflict with the observations (2), (3) and (4) and do not 15 allow for a direct transfer into CDD. The development of a continuum theory of dislocation segments connecting different slip systems, which would be needed to address those observations, is complex and beyond the scope of this paper. However, we address the observations (1) and (2) in conjunction with the available kinematics of the CDD formulation.

The expansion of existing dislocations yielding a dislocation density increase, i.e. an increase of the dislocation line length, is incorporated in CDD via the source term $v^{\xi} q^{\xi}$ in the dislocation density evolution Eq. (3). The increase in dislocation density is thereby due to the expansion of an existing curved dislocation. However, the total angular content, namely the number of dislocations in the system determined by the integral of $q^{\xi}$, is conserved and the evolution equations of all internal variables are restricted to the individual slip system. Dislocation multiplication

25 in DDD by contrast is always connected to a change of dislocation segments between slip systems and a subsequent expansion of the newly generated dislocations. Thereby, the physical dislocation line, which initiated the multiplication process transforms into virtual line length, but remains a part of the involved dislocation loop. Further, the concentration of curvature in the end-nodes of the multiplication object reduces the ability of the constituting dislocations to ${ }_{30}$ increase their line length by their further expansion, which can be interpreted as a reduction in 'potential' of dislocation expansion. In order to achieve a dislocation multiplication in line with the DDD observations, the increase of the angular content in the system based on the transfer of dislocation density between slip systems and an inhibition of the expansion of dislocations, which initiate multiplication has to be included in CDD. Therefore, it is necessary to formulate 35 equations, which include both the dislocation density and the curvature density. In the sequel we shall reserve the term dislocation multiplication for the generation of new dislocation density and curvature density in contrast to the mere expansion of existing dislocations.

In the following, we present a model of dislocation multiplication, which homogenizes the multiplication mechanisms observed in DDD [1]. Each mechanism contributes to multiplication

40 with its individual rate at which dislocation density and curvature density is generated on a slip system at the expense of dislocation density on one (in case of cross-slip), or two (in case of glissile reactions) other slip systems. This means a generation of closed dislocation loops, which can evolve according to the existing kinematics. However, the current formulation of the CDD kinematics does not permit a concentration of the curvature in a single point as in DDD. Further,

45 the concept of a 'virtual dislocation line' does not exist in CDD. We thus account for the coupling of the slip systems and the limitation of the expansion of the dislocations by reducing dislocation density and curvature density on the slip systems, which initiate the multiplication process. 


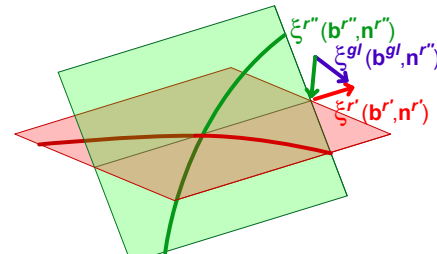

(a) Intersection of curved dislocation lines.

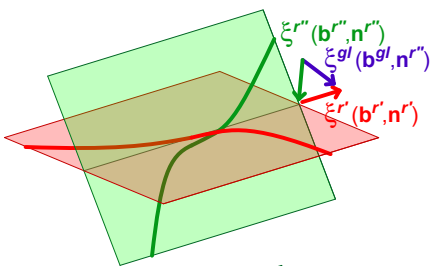

(b) Alignment of dislocation lines.

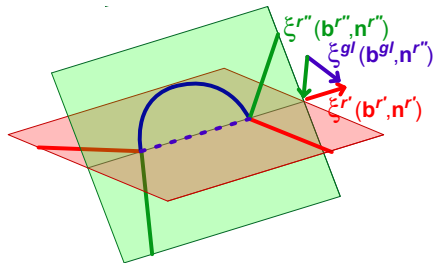

(c) Formation of the glissile junction (dotted blue line) and its bow-out under stress (solid blue line).

Figure 1: Schematic visualization of the formation of a glissile reaction between two intersecting curved dislocation lines on the slip systems $\xi^{r^{\prime}}$ and $\xi^{r^{\prime \prime}}$ with Burgers vector $\mathbf{b}$ and slip plane normal $\mathbf{n}$ (a). The reaction leads to an alignment of the dislocation lines due to elastic interaction (b) and subsequently to the formation of a dislocation junction on the glissile system $\xi^{g l}$ (dotted blue line). The dislocation junction lies on the same plane as the dislocation on the reacting slip system $\xi^{r^{\prime \prime}}$ and is able to bow-out under stress (solid blue line) (c). The reaction leads to a concentration of the dislocation curvature on the reacting slip systems $\xi^{r^{\prime}}$ and $\xi^{r^{\prime \prime}}$ into the endpoints of the junction.

\subsubsection{Dislocation multiplication due to glissile reactions}

As a first mechanism, we consider the dislocation multiplication due to glissile reactions, which have the highest probability of occurrence in fcc crystals according to [23]. To point out the interplay of slip systems by the glissile reaction, we first derive the density equations on

5 one exemplary reaction using the superscripts $r^{\prime}, r^{\prime \prime}$ and $g l$ for the involved slip systems. The formulations are largely based on observations in the DDD simulations in [1] and recast into a homogenized continuum description. The process of the glissile reaction formation in DDD is schematically shown in Fig. 1. For simplicity, the visualization shows a dislocation line segment of one dislocation loop on each slip system before the lines intersect (Fig. 1(a)). However, in the

10 continuum theory we assume that generally only a statistical number of dislocations are affected by the reaction in each continuum element. In DDD, after an alignment of the dislocation lines due to the elastic interaction, shown in Fig. 1(b), two reaction partners on the slip systems $\xi^{r^{\prime}}$ and $\xi^{r^{\prime \prime}}$ react and form a mobile dislocation on a third slip system $\xi^{g l}$. This implies the formation of a dislocation junction on the glissile system $\xi^{g l}$ (dotted blue line in Fig. 1(c)) and its expansion 5 under stress (solid blue line in Fig. 1(c)) at the expense of the involved dislocation line length on the reacting slip systems $\xi^{r^{\prime}}$ and $\xi^{r^{\prime \prime}}$. In the continuum model, we do not capture the exact process of the glissile reaction formation, but rather generate a new dislocation with the appropriate curvature and line length. We incorporate the evolution equations proposed in [23] formulating the dislocation density generation. There, the junction formation rate is derived by calculating a collision frequency between an active system $\xi$ and a forest system $\zeta$ as

$$
\nu^{\xi}=\frac{v^{\xi}}{L^{\zeta}} \quad \text { with } \quad \xi, \zeta=r^{\prime}, r^{\prime \prime} \quad \text { and } \quad \xi \neq \zeta
$$

using the average dislocation spacing of

$$
L^{\zeta} \propto \frac{1}{\sqrt{\rho^{\zeta}}} .
$$

A dislocation line on each of the reacting slip systems can be both active or act as a forest density. Therefore the rate of dislocation density generation on the glissile system $\xi^{g l}$ - without 


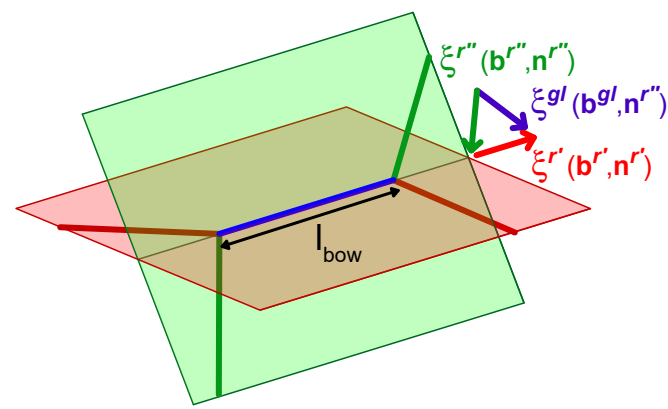

(a) $\left|v^{g l}\right|=0$.

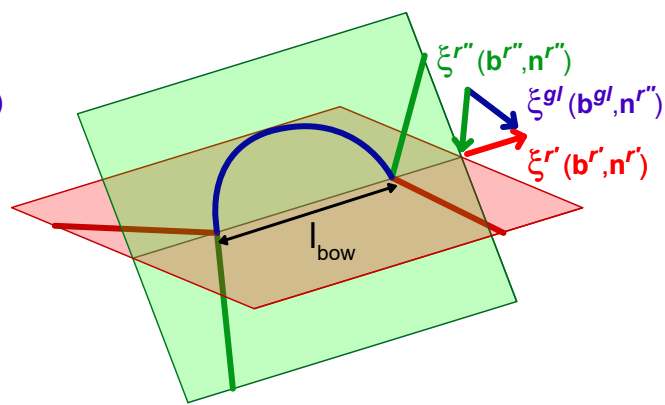

(b) $\left|v^{g l}\right|>0$.

Figure 2: Configuration of the newly generated dislocation loop on the glissile system $\xi^{g l}$ for $\left|v^{g l}\right|=0$, leading to a dislocation deposition for $\left|v^{g l}\right|=0$ (a) and $\left|v^{g l}\right|>0$, leading to a bow-out for $\left|v^{g l}\right|>0$ (b).

considering the bow-out - consist of two parts given as

$$
\partial_{t} \rho_{\text {gliss }}^{g l} \propto \rho^{r^{\prime}} \nu^{r^{\prime}}+\rho^{r^{\prime \prime}} \nu^{r^{\prime \prime}}
$$

where the ()$_{\text {gliss }}$ stands for the glissile mechanism. Thus holds

$$
\partial_{t} \rho_{\text {gliss }}^{g l}=C_{1}\left(\rho^{r^{\prime}} v^{r^{\prime}} \sqrt{\rho^{r^{\prime \prime}}}+\rho^{r^{\prime \prime}} v^{r^{\prime \prime}} \sqrt{\rho^{r^{\prime}}}\right),
$$

where the constant $C_{1}$ depends on the magnitude of the Burgers vector and the effective junction length [23]. The order of magnitude of $C_{1}$ has been determined by DDD simulations [6].

In DDD, a bow-out can follow the junction formation, which leads to an increase of line length and curvature on the glissile system $\xi^{g l}$, shown by the solid blue line in Fig. 1(c). Most of the newly generated dislocations show a motion of the shared end-nodes of the junction along the intersecting line of the respective slip planes, which greatly reduces the stress needed to move the dislocation line [1]. In the available CDD formulation, there is no representation of the effect of 10 sliding end-nodes of dislocation junctions coupling different slip systems. However, we assume that the average distance between junction points scales with the mean dislocation spacing,

$$
l_{\text {bow }}=\frac{1}{\sqrt{\rho}}
$$

which we take to be the same on all slip systems. We consequently choose the average dislocation spacing as local average bow-out length which is in accordance with [1].

Fig. 2 schematically visualizes the dislocation configurations generated by the bow-out for

15 zero and non-zero velocity on the glissile system $\xi^{g l}$, incorporating the bow-out length $l_{\text {bow. }}$. In case of a zero velocity on the glissile system, we assume the generation of line length without a generation of curvature (Fig. 2(a)). However, in case of non-zero velocity on the glissile slip system, which is the general case under stress, the glissile reaction is assumed to generate a new dislocation with an increasing line length and given curvature due to the bow-out (Fig. 2(b)).

20 Thus, we see the bow-out in case of non-zero velocity as part of the process, which generates a new dislocation.

Using this distinction, we denote the contribution of the bow-out to the generated dislocation density on the glissile system $\xi^{g l}$ by the newly generated dislocation density $\rho_{\mathrm{m}, \mathrm{gliss}}^{g l}$ and the 
generated curvature density $q_{\mathrm{m} \text {,gliss }}^{g l}$. Here, a subscript () is used to indicate the densities, which are a result of the dislocation multiplication process. The density $\rho_{\mathrm{m}, \mathrm{gliss}}^{g l}$ includes at least the dislocation density, which is generated on the glissile system $\xi^{g l}$ without bow-out, i.e. $\rho_{\text {gliss }}^{g l}$ in Eq. (13). In case of a zero velocity, thus it holds $\partial_{t} \rho_{\mathrm{m} \text {,gliss }}^{g l}=\partial_{t} \rho_{\text {gliss }}^{g l}$ and $\partial_{t} q_{\mathrm{m}, \mathrm{gliss}}^{g l}=0$, compare 5 the schematic sketch in Fig. 2(a). In any other case, i.e. $v^{g l} \neq 0$, the bow-out results in a generation of a dislocation with increased line length and curvature density proportional to the mean dislocation spacing (Eq. 14), schematically shown in Fig. 2 (b). Since the dislocation can only bow-out in one direction and according to [1] almost never completely detaches from its initial site, we assume an average upper limit of the dislocation density production based on the

10 line length of a half-circle with the circumference of $\frac{\pi}{2} l_{\text {bow }}$. In DDD, most of the dislocation lines are immobilized in the dislocation network, or the dislocation does not reach the halfcircle state because the shared end-nodes of the junction are moving apart. Since the current kinematic framework of CDD does not allow for an exact reproduction of this effect, we do not aim to capture the bow-out process itself. Instead, we account for the characteristics of the

15 dislocation network to limit the extent of the bow-out by a parameter $C_{2}$. With this assumption, the resulting rate of dislocation density generation accounting for the bow-out of the dislocations can be modeled by

$$
\partial_{t} \rho_{\text {m,gliss }}^{g l}= \begin{cases}C_{2} \frac{\pi}{2} \partial_{t} \rho_{\text {gliss }}^{g l} & \text { if }\left|v^{g l}\right|>0 \\ \partial_{t} \rho_{\text {gliss }}^{g l} & \text { if }\left|v^{g l}\right|=0 .\end{cases}
$$

We describe the average radius of the generated dislocations by the relation $l_{\text {bow }} / 2=\partial_{t} \rho_{\mathrm{m}, \mathrm{gliss}}^{g l} / \partial_{t} q_{\mathrm{m}, \mathrm{gliss}}^{g l}$ using the average bow-out length $l_{\text {bow }}$ from Eq. (14). Thus we obtain the rate of curvature density 20 generation as

$$
\partial_{t} q_{\mathrm{m}, \mathrm{gliss}}^{g l}=\operatorname{sign}\left(v^{g l}\right) C_{2} \pi \partial_{t} \rho_{\mathrm{gliss}}^{g l} \sqrt{\rho} .
$$

Since in case of $v^{g l}<0$ dislocation loop expansion is caused by negative curvature density (see Eq. 3), we assume $\partial_{t} q_{\mathrm{m} \text {,gliss }}^{g l}<0$ if $v^{g l}<0$. In the current analysis, we choose $C_{2}=1$, which implies that the bow-out under stress on average expands up to half of the mean dislocation spacing, as shown in Fig. 2(b). This value can change, e.g. by accounting for the sliding end25 nodes of dislocation junctions.

By generating curvature density in along with the dislocation density, the multiplication model not only increases the line length on the glissile system, but also generates new dislocations in the sense of new closed objects, which is the process we call dislocation multiplication. These new dislocations subsequently can move according to the existing kinematics and 30 increase their line length due to loop expansion until the dislocations are involved in a similar multiplication event as they are generated from.

The DDD simulations show, that dislocation reactions lead to a loss of line length on the reacting slip systems $\xi^{r^{\prime}}$ and $\xi^{r^{\prime \prime}}$ in favor of line length, which is part of the dislocation junction [1]. Furthermore, the reaction limits the expansion of the involved dislocations on the reacting

35 slip systems. To account for the loss of line length on the slip systems $\xi^{r^{\prime}}$ and $\xi^{r^{\prime \prime}}$ (schematically visualized by the dotted blue line in Fig. 1(c)), an annihilation rate is included on the reacting slip systems

$$
\partial_{t} \rho_{\text {gliss }}^{\xi}=-C_{3} \partial_{t} \rho_{\text {gliss }}^{g l} \quad \text { with } \quad \xi=r^{\prime}, r^{\prime \prime},
$$

where we use Eq. (13) including a parameter $C_{3}$ scaling the loss of line length on the reacting slip systems with the line length of the generated dislocation on the glissile system. Thereby, each of 40 the glissile reactions leads to a generation of a mobile dislocation on a glissile system (Eq. 15 and 
13) at the expense of the two reacting slip systems. The reaction will therefore lead to a decrease of dislocation density on average for $C_{3}>\frac{\pi}{4} C_{2}$. In the following analysis, we choose $C_{3}=1$ as simplistic approximation and upper limit of the dislocation density reduction. A consideration of $C_{3}=\frac{\pi}{4} C_{2}$ as lower limit such that no dislocation density is annihilated for a bowed out junction 5 is analyzed in Appendix A.

In the CDD theory, the total dislocation density in one element contains a certain angular content, which is represented in the curvature density $q^{\xi}$. Thus by removing a part of the dislocation density, a certain angular range is removed as well. We homogenize this process by formulating the reduction in curvature density by the dislocation reaction on each of the reacting 10 slip systems $\xi^{r^{\prime}}$ and $\xi^{r^{\prime \prime}}$ as proportional to the reduction in line length. The reduction in curvature density can then be derived as

$$
\partial_{t} q_{\text {gliss }}^{\xi}=-\frac{\partial_{t} \rho_{\text {gliss }}^{g l}}{\rho^{\xi}} q^{\xi} \quad \text { with } \quad \xi=r^{\prime}, r^{\prime},
$$

where $\rho^{\xi}$ and $q^{\xi}$ are the dislocation density and curvature density on the reacting slip system.

In DDD, the dislocation network mostly consists of rather straight dislocation lines, which start and end in dislocation junctions or at the surface. Dislocations typically do not expand 5 any further after taking place in multiplication events on reacting slip systems. The curvature (angular change) is observed to be equilibrated at the end-nodes of dislocation junctions due to the dislocation network [1]. In CDD, an existing curvature density on the reacting slip systems always leads to additional line length production in non-zero resolved shear stress conditions. As a consequence, the immobilizing effect observed in DDD can therefore not be captured by

20 the average curvature density. A formulation, which approximates this effect in CDD can currently only be achieved by assuming that the dislocation curvature does not further contribute to the source term $v^{\xi} q^{\xi}$ in the evolution equation of the dislocation density (Eq. 3). Thereby the 'potential' of line length increase by dislocation loop expansion is reduced, mimicking the immobilizing effect of the junction on its constituting dislocations. We apply this assumption

25 to the dislocations, which take part in the multiplication event on the reacting slip systems $\xi^{r^{\prime}}$ and $\xi^{r^{\prime \prime}}$, as shown in Fig. 1(c) in an exemplary and simplified way by straight lines. This is a second contribution to the reduction in curvature density on the reacting slip systems $\xi^{r^{\prime}}$ and $\xi^{r^{\prime \prime}}$, which temporarily slows down the generation of dislocation density by expansion of existing dislocation loops in the given time step.

30 For simplicity, we assume that the reduction in curvature density is proportional to the reduction in line length due to the reaction, which results in the same relation as derived above (Eq. 18), assuming a reduction in curvature with constant line length. However, in general the reduction of curvature density depends on the type of multiplication mechanism (glissile reaction or cross-slip) and dislocation configuration. Therefore, the scaling between the curvature den-

35 sity reduction and the reduction of dislocation density is considered by a parameter $C_{4}$. For the reduction of curvature density, we assume two contributions, which are schematically depicted in Fig. 1(c): First, a contribution from the reduction of line length (dotted blue line) and second, a contribution from the reduction of 'potential' for line length increase by dislocation loop expansion (remaining straight dislocation lines on slip systems $\xi^{r^{\prime}}$ and $\xi^{r^{\prime \prime}}$ ).

By combining both contributions, we derive the final formulation for the reduction of curvature density on the reacting slip systems $\xi^{r^{\prime}}$ and $\xi^{r^{\prime \prime}}$ due to the multiplication event as

$$
\partial_{t} q_{\text {gliss }}^{\xi}=-C_{4} \frac{\partial_{t} \rho_{\text {gliss }}^{g l}}{\rho^{\xi}} q^{\xi} \quad \text { with } \quad \xi=r^{\prime}, r^{\prime} .
$$


In the current analysis, we choose $C_{4}=2$ by assuming, that both contributions are added and are directly proportional to the reduction in line length.

\subsubsection{Dislocation multiplication by cross-slip}

As a second multiplication mechanism, we consider the dislocation multiplication by cross5 slip, which incorporates the generation of dislocation density on the cross-slip system $\xi^{c s}$ based on the difference in shear stress between the primary slip system $\xi^{p}$ and its cross-slip system $\xi^{c s}$. The formulation of a generalized cross-slip model for use in different microstructure configurations requires the transformation of the probabilistic nature of cross-slip [28, 29, 30] into a homogenized continuum theory. In the interest of clarity and the objective of the current study,

10 we present a simple model based on the cross-slip implementation in the underlying DDD simulations [1]. In this subsection, we derive the evolution equations for the dislocation densities using the superscript $p$ for the primary slip system and $c s$ for the cross-slip system. For the cross-slip to take place, we assume that the resolved shear stress on the cross-slip system $\xi^{c s}$ has to be equal or greater than the resolved shear stress on the primary slip system $\xi^{p}$, i.e. $\left|\tau^{c s}\right| \geq\left|\tau^{p}\right|$.

15 We ignore slip systems with zero shear stress, thus we apply the condition that cross-slip only occurs if $\tau^{p}, \tau^{c s} \neq 0$. We introduce a thermally activated cross-slip probability, which is similar to the probability term used in DDD-models, e.g. [28, 29], as

$$
P\left(L^{p}\right)=\min \left\{\beta \frac{L^{p}}{L_{0}} \frac{\Delta t}{\Delta t_{0}} \exp \left(\frac{V_{\text {act }}}{k_{\mathrm{B}} T}\left(\left|\tau^{c s}\right|-\tau_{\mathrm{III}}\right)\right) ; 1\right\},
$$

where $L^{p}$ is the length of the dislocation line on the primary slip system, which is able to crossslip, $\tau^{c s}$ is the resolved shear stress on the cross-slip system and $\Delta t$ is the time-step used in

20 the simulation. The other parameters are the same as in the underlying DDD simulations: A normalization constant $\beta=10^{5}$, the activation volume $V_{a c t}=300 b^{3}$, the shear stress at the onset of stage III hardening $\tau_{\mathrm{III}}=5 \mathrm{MPa}$ and a reference value for length $L_{0}=1 \mu \mathrm{m}$ and time $\Delta t_{0}=1 \mathrm{~s}$ $[29,30] . k_{B}$ is the Boltzmann constant and the temperature $T$ is chosen to $293 \mathrm{~K}$.

As suggested in [30], we assume that dislocation lines within $\pm 10^{\circ}$ of the ideal screworientation are able to cross-slip. In order to model the fraction of density which is able to cross slip, we need assumptions on how the dislocation density is distributed on the circle of directions in the slip plane. For this we employ the assumption that the SSD-density, i.e. $\rho-\left\|\boldsymbol{\kappa}^{p}\right\|$, is homogeneously distributed among all line directions. Thus, $1 / 9$ of the SSD density $\left(20^{\circ}\right.$ around the positive and negative screw direction each) is potentially able to cross-slip. For the GNDs we

so assume that they are potentially able to cross-slip, if the GND orientation lies within $\pm 10^{\circ}$ of the positive or negative screw-orientation. The absolute value of the cosine of the angle the GND vector includes with the screw direction is given by $\frac{\left|\tilde{\kappa}^{p}\right|}{\left\|\kappa^{p}\right\|}$, where $\tilde{\kappa}^{p}$ denotes the screw component of the GND vector. The possibility of GNDs to cross slip may therefore be derived by evaluating the Heaviside step function $\Theta\left(\frac{\left|\tilde{R}^{p}\right|}{\mid \kappa^{p} \|}-\cos \left(\frac{\pi}{18}\right)\right)$, which equals 1 if the GND orientation lies within

$35 \pm 10^{\circ}$ of the screw orientations and 0 otherwise. To obtain the amount of GND density which may potentially cross slip the result of the Heaviside function is multiplied by $\left\|\boldsymbol{\kappa}^{p}\right\|$. The total density which is capable of cross-slip on the primary slip system $\xi^{p}$ including the contribution of the SSD and GND-density is consequently calculated as

$$
\tilde{\rho}^{p}=\frac{1}{9}\left(\rho^{p}-\left\|\boldsymbol{\kappa}^{p}\right\|\right)+\left\|\boldsymbol{\kappa}^{p}\right\| \Theta\left(\frac{\left|\tilde{\kappa}^{p}\right|}{\left\|\boldsymbol{\kappa}^{p}\right\|}-\cos \left(\frac{\pi}{18}\right)\right) .
$$

During the review process one of the reviewers made us aware that the current model introduces a 
results this discontinuity did not cause discernible problems. However, in Appendix B, we briefly describe how this discontinuity may be avoided, based on a suggestion of the very reviewer.

The length of the dislocation line, which is able to cross-slip is determined from the average dislocation loop radius $r^{p}=\rho^{p} / q^{p}$ in an averaging volume

$$
L^{p}=\min \left\{\frac{2 \cdot 10^{\circ}}{360^{\circ}} 2 \pi r^{p} ; L\right\}
$$

5 where we limit the dislocation line length to the local mean dislocation spacing $L=\frac{1}{\sqrt{\rho}}$, which we assume to be the same for all slip systems.

Using the probability term Eq. (20), the production rate of the density which cross-slips to the respective cross-slip system $\xi^{c s}$ is

$$
\partial_{t} \rho_{\mathrm{cross}}^{c s}=\tilde{\rho}^{p} \frac{P\left(L^{p}\right)}{\Delta t},
$$

the production rate on the primary slip system $\xi^{p}$, which loses the respective dislocation density 10 is

$$
\partial_{t} \rho_{\text {cross }}^{p}=-\partial_{t} \rho_{\text {cross }}^{c s} .
$$

We assume that the screw GNDs cross-slip similar to the total density, which means that screw GND density keeps its orientation after cross-slip and does not transform into SSDs. Thus, the same relation as for the total dislocation density holds for the screw GND-density for both involved slip systems

$$
\begin{aligned}
& \partial_{t} \tilde{\kappa}_{\text {cross }}^{c s}= \begin{cases}\frac{P^{p}\left(L^{p}\right)}{\Delta t}\left\|\boldsymbol{\kappa}^{p}\right\| \Theta\left(\frac{\left|\tilde{\boldsymbol{K}}^{p}\right|}{\left\|\boldsymbol{K}^{p}\right\|}-\cos \left(\frac{\pi}{18}\right)\right) & \text { if } \tilde{\boldsymbol{\kappa}}^{p}>0 \\
-\frac{P{ }^{p}\left(L^{p}\right)}{\Delta t}\left\|\boldsymbol{\kappa}^{p}\right\| \Theta\left(\frac{\left|\tilde{\kappa}^{p}\right|}{\left\|\boldsymbol{K}^{p}\right\|}-\cos \left(\frac{\pi}{18}\right)\right) & \text { if } \tilde{\boldsymbol{\kappa}}^{p}<0\end{cases} \\
& \partial_{t} \tilde{\kappa}_{\text {cross }}^{p}=-\partial_{t} \widetilde{\kappa}_{\text {cross }}^{c s} .
\end{aligned}
$$

15 The curvature density part of the multiplication process through cross-slip is formulated according to Eqs. $(18,19)$. The contribution of the bow-out to the increase of dislocation density and curvature density is considered analogously to Eqs. $(15,16)$ by the contribution of the rate of dislocation density generation $\partial_{t} \rho_{\mathrm{m}, \text { cross }}^{c s}$ and curvature density generation $\partial_{t} q_{\mathrm{m}, \text { cross }}^{c s}$ to multiplication as

$$
\partial_{t} \rho_{\mathrm{m}, \mathrm{cross}}^{c s}=\frac{\pi}{2} \partial_{t} \rho_{\mathrm{cross}}^{c s}
$$

20 and

$$
\partial_{t} q_{\mathrm{m}, \mathrm{cross}}^{c s}=\operatorname{sign}\left(v^{c s}\right) \pi \partial_{t} \rho_{\mathrm{cross}}^{c s} \sqrt{\rho} .
$$

Since the relations presented in Eqs. (20 - 27) hold also for the reversed order of the primary and the cross-slip system, slip systems with the same shear stress show equal dislocation density generation as long as the density is the same.

\subsubsection{Combined mechanisms of dislocation density}

Combining the multiplication mechanisms described in section 2.2.1 and 2.2.2, we incorporate the dislocation density generation and reduction due to glissile reactions and cross-slip, 


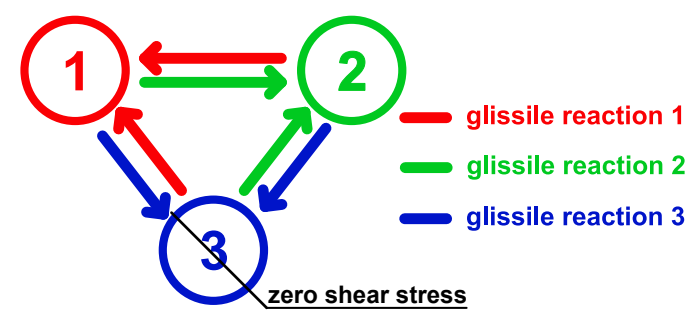

Figure 3: schematic overview of the three considered slip systems $\xi=1,2,3$ with the respective glissile reactions.

which affects line length and curvature density, into the CDD evolution equations, see Eq. (3). The extended evolution equations are given as

$$
\begin{aligned}
& \partial_{t} \rho^{\xi}=-\nabla \cdot\left(v^{\xi} \boldsymbol{\kappa}_{\perp}^{\xi}\right)+v^{\xi} q^{\xi}+\partial_{t} \rho_{\mathrm{m}}^{\xi}+\partial_{t} \rho_{\mathrm{gliss}}^{\xi}+\partial_{t} \rho_{\text {cross }}^{\xi} \\
& \partial_{t} \boldsymbol{\kappa}^{\xi}=\nabla \times\left(\rho^{\xi} v^{\xi} \mathbf{m}^{\xi}\right)+\partial_{t} \boldsymbol{\kappa}_{\text {cross }}^{\xi} \\
& \partial_{t} q^{\xi}=-\nabla \cdot\left(\frac{q^{\xi}}{\rho^{\xi}} \boldsymbol{\kappa}_{\perp}^{\xi} v^{\xi}+\boldsymbol{A}^{\xi} \nabla v^{\xi}\right)+\partial_{t} q_{\mathrm{m}}^{\xi}+\partial_{t} q_{\mathrm{gliss}}^{\xi}+\partial_{t} q_{\text {cross }}^{\xi},
\end{aligned}
$$

where $\partial_{t} \boldsymbol{\kappa}_{\text {cross }}^{\xi}=\left(\partial_{t} \tilde{\kappa}_{\text {cross }}^{\xi}, 0,0\right)$. Each slip system $\xi$ contains a reduction in dislocation density and curvature density due to glissile reactions and cross-slip as well as an increase due to generation of dislocations including the bow-out. The individual contributions from dislocation generation through glissile reactions and cross-slip are combined into one variable each for the rate of dis5 location density generation $\partial_{t} \rho_{\mathrm{m}}^{\xi}=\partial_{t} \rho_{\mathrm{m}, \text { gliss }}^{\xi}+\partial_{t} \rho_{\mathrm{m}, \text { cross }}^{\xi}$ and the curvature density generation $\partial_{t} q_{\mathrm{m}}^{\xi}=\partial_{t} q_{\mathrm{m}, \mathrm{gliss}}^{\xi}+\partial_{t} q_{\mathrm{m}, \mathrm{cross}}^{\xi}$

\subsection{Simulation setups}

\subsubsection{Simplified systems}

In order to illustrate the presented multiplication mechanisms in the continuum framework,

10 we first neglect all dislocation density fluxes in the set of equations Eq. (28) to solely evaluate the multiplication terms in a simple benchmark setup. In this local continuum formulation all spacial gradients in the evolution equations are zero, resulting in a system of ordinary differential equations. The setup mimics a fully periodic system, in which no dislocation lines can leave the simulation volume. Although this configuration is artificial, it allows to investigate the behavior

15 of the equations of the multiplication model in a setup, which is not influenced by the different orientation of the individual slip systems with respect to the system geometry. We analyze the influence of the glissile and the cross-slip mechanism separately. The elastic material parameters are given by the elastic modulus $E=71.3 \mathrm{GPa}$, a Poisson's ratio $\nu=0.34$ and a Burgers vector of $b=0.256 \mathrm{~nm}$.

${ }_{20}$ First, we only consider the glissile mechanism as described in section 2.2.1. We restrict the investigation to three slip systems incorporating three glissile reactions, as schematically shown in Fig. 3. Two slip systems $(\xi=l$ and $\xi=2)$ are subjected to a constant shear stress on the slip system of $\tau_{1, \text { ext }}=\tau_{2, \text { ext }}=15 \mathrm{MPa}$, which resembles a resolved shear stress due to external loading. On slip system $\xi=3$ the shear stress is set to zero $\left(\tau_{3, \mathrm{ext}}=0\right)$ in order to mimic active

25 and inactive slip systems. We choose an initial dislocation density of $\rho_{1}=\rho_{2}=3 \times 10^{12} \frac{1}{m^{2}}$ on the active slip systems 1 and 2 and $\rho_{3}=1 \times 10^{12} \frac{1}{m^{2}}$ on the inactive slip system 3. Furthermore, 


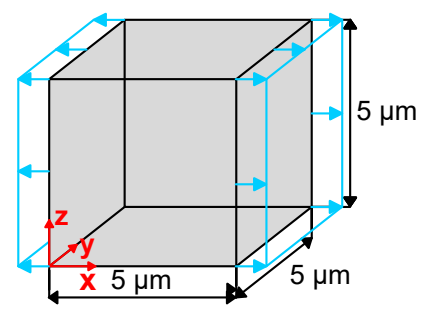

Figure 4: System setup for CDD - DDD comparison.

the initial dislocation density vector and the initial curvature are set to zero, $\boldsymbol{\kappa}^{\xi}=0$ and $q^{\xi}=0$. The glissile constant $C_{1}$ is chosen as $C_{1}=0.064$ similar to [6]. This yields a configuration in which the dislocation density on active slip systems is larger than on the inactive slip system, but all slip systems are a possible partner of the glissile reactions.

In a second example, we only evaluate the cross-slip mechanism as explained in section 2.2.2. We consider a system with two slip systems $(\xi=1$ and $\xi=2)$ subjected to two different stress configurations: (i) Both slip systems have the same shear stress $\tau_{1, \mathrm{ext}}=\tau_{2, \mathrm{ext}}=15 \mathrm{MPa}$ and (ii) the slip systems have different shear stresses of $\tau_{1, \text { ext }}=10 \mathrm{MPa}$ and $\tau_{2, \text { ext }}=15 \mathrm{MPa}$. In both stress configurations, we choose a homogeneous initial dislocation density of $\rho_{1}=\rho_{2}=1 \times 10^{12} \frac{1}{\mathrm{~m}^{2}}$ with $\boldsymbol{\kappa}^{\xi}=0$ and $q^{\xi}=0$.

\subsubsection{Full fcc system with tensile loading}

We aim to compare the presented multiplication model to DDD simulations from [1]. Therefore, we apply the model to a cubic system as shown in Fig. 4 with edge length of $5 \times 5 \times 5 \mu \mathrm{m}$ and 12 fcc slip systems.

15 The system is subjected to a constant strain-rate of $\dot{\varepsilon}=5000 \mathrm{~s}^{-1}$ by applying a tensile displacement on the $x$-normal-surfaces in $x$-direction aligned with the [100] crystal orientation. The side surfaces are traction free. Dislocation lines can leave the simulation volume through all surfaces. This is achieved by allowing for an outflow of dislocation density flux in direction of the outer surface normal vector. A detailed explanation of the numerical implementation is given in

20 [31]. In the configuration investigated here, there are eight slip systems with the same nonzero Schmid-factor, i.e. active slip systems, and four with a zero Schmid-factor, i.e. inactive slip systems. For the latter, we set $v=0$ in order to avoid dislocation motion and cross-slip activity on these systems due to stress concentrations at the external boundaries. This ensures, that any dislocation evolution observed on inactive slip systems is only caused by glissile reactions. The

25 strain-rate and boundary conditions for the dislocation density fluxes are chosen corresponding to the DDD simulations from [1].

In the DDD simulations, the dislocation structure is relaxed from a random distribution of closed dislocation loops prior to straining [1]. This leads to the formation of a dislocation network, which shows a certain density distribution in the inner region and declines towards the

so boundaries, as shown for one representative DDD simulation in Fig. 5(a). Due to the random initial loop distribution, the topology of each dislocation network is slightly different. However, the higher dislocation population in the inner region of the system is a common characteristic of all relaxed dislocation networks. In the continuum, we mimic this characteristic by prescribing a SSD density distribution in the center of the system, which decreases towards the boundaries 35 as shown in Fig. 5(b). The GND density is assumed to be zero in the initial state. The decrease 


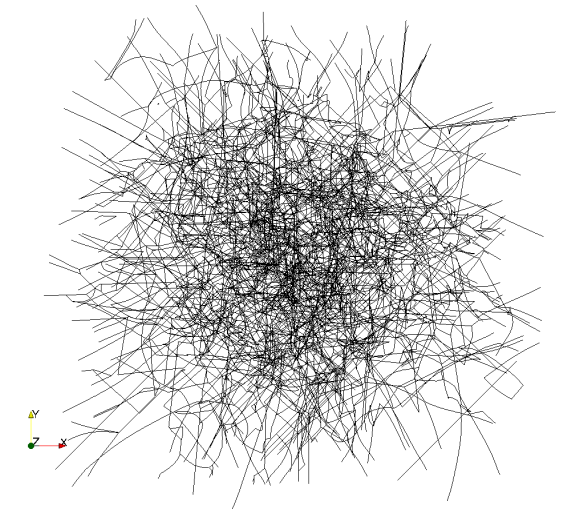

(a) Relaxed initial dislocation microstructure of one representative DDD simulation.

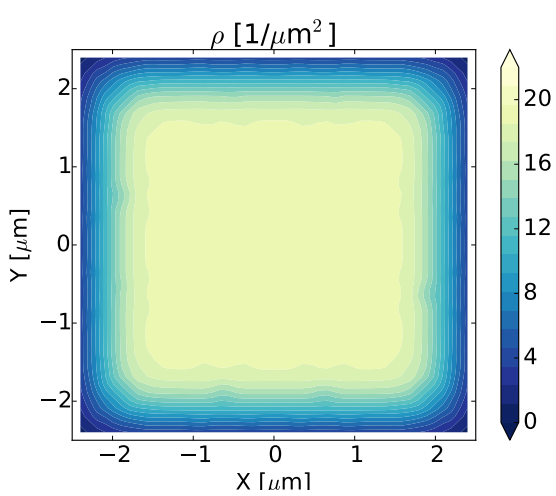

(b) Initial total dislocation density distribution in CDD.

Figure 5: Relaxed initial dislocation microstructure in one representative DDD simulation (a) and initial distribution of the total dislocation density in CDD in a central plane averaged over all slip systems (b).

of the dislocation density at the boundaries is described by a cumulative distribution function of the Gaussian normal distribution (Gaussian error function) with a gradient towards the outer boundaries in the three coordinate directions. For the Gaussian error function we use a mean value of $2.15 \mu \mathrm{m}$ and a standard deviation of 0.3 to distribute an initial dislocation density of $5 \rho^{\xi}=1.6 \times 10^{12} \frac{1}{m^{2}}$ per slip system in the middle of the system to almost zero at the boundaries. This results in an average density of $\rho^{\xi} \approx 1 \times 10^{12} \frac{1}{m^{2}}$ per slip system and a total initial dislocation density of $\rho_{\text {tot }} \approx 1.19 \times 10^{13} \frac{1}{m^{2}}$, when summarized over all slip systems. We chose $\boldsymbol{\kappa}^{\xi}=0$ and $q^{\xi}=0$ initially in order to focus on the interplay of the dislocation density between slip systems. The elastic material parameters and the constant $C_{1}$ are chosen as before.

\section{Results}

In this section, we first consider the simplified system setup described in section 2.3.1 to understand the general behavior of the set of equations (Eq. 28) for glissile and cross-slip individually. Then, we apply the multiplication model to the setup including $12 \mathrm{fcc}$ slip systems and compare the results to DDD simulations.

15 3.1. Investigation of multiplication mechanisms in simplified slip configurations

\subsubsection{Glissile reactions in a simplified system}

In a first analysis, we only consider glissile reactions occurring between three slip systems, where the shear stress is zero on slip system 3, as visualized in Fig. 3. Therefore, the slip system 3 is inactive and serves only as a passive reaction partner, which also contributes to the total dislocation density evolution according to Eq. (13). We assume an infinite homogeneous system such that all spatial gradients can be neglected in Eq. (28), resulting in a system of ordinary differential equations. The evolution of the dislocation density per slip system over simulation time is depicted in Fig. 6(a). The glissile reactions between the slip systems yield an increase in dislocation density on the active as well as the inactive slip systems until a saturation after about $0.4 \mu$ s with no significant further increase in dislocation density. On the two active slip systems, 


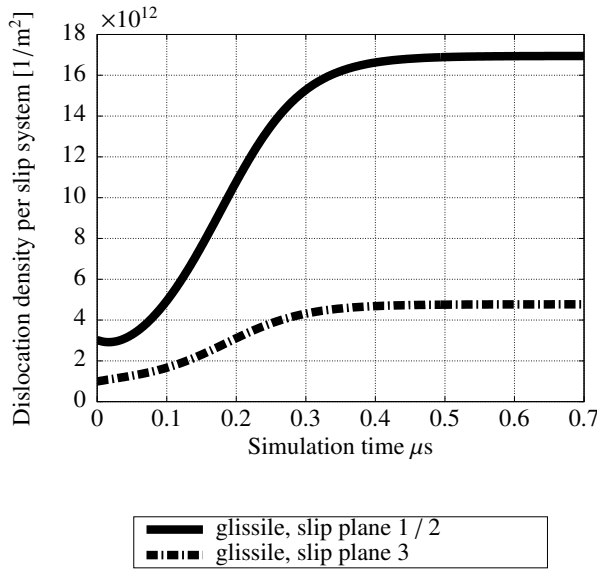

(a) Dislocation density per slip system with glissile reactions.

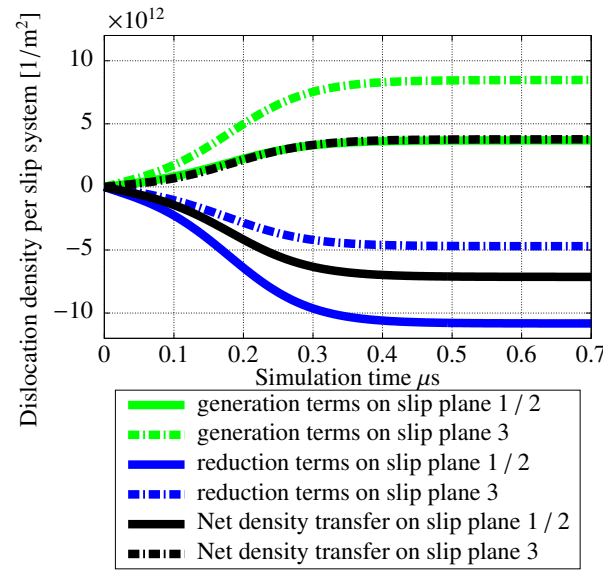

(b) Contribution of the glissile evolution equation.

Figure 6: Dislocation density per slip system over simulation time (a) and contribution of the glissile evolution equation by the dislocation density reduction and density generation terms integrated over the simulation time (b) for the simplified setup in section 2.3.1. Additionally, the net density transfer on each slip system is shown.

a slight decrease in dislocation density is observed directly at the beginning of the simulation. The final saturation of the dislocation density is caused by the increase of the yield stress with increasing dislocation density, which reaches the applied shear stress (Eq. 6), rendering all slip systems inactive.

To further investigate the observed dislocation density behavior, the contribution of the glissile evolution equations in Eq. (28) are compared separately for the active slip systems 1 and 2 and the inactive slip system 3 in Fig. 6(b). Each of the slip systems is involved in two individual reactions with a second slip system, see also Fig. 3. First, it contributes to a reduction in dislocation density $\left(\partial_{t} \rho_{\text {gliss }}^{\xi}\right)$. Second, new dislocation density is generated due to the reac-

10 tion of the other two slip systems, contributing to a dislocation density increase $\left(\partial_{t} \rho_{\mathrm{m}, \mathrm{gliss}}^{\xi}\right)$. By summarizing the density reduction and density generation on each slip system, the net density transfer between the slip systems is obtained. The rates are integrated over time given as $\rho_{\text {gliss }}^{\xi}=\int_{t} \partial_{t} \rho_{\text {gliss }}^{\xi} d t$ and $\rho_{\text {m,gliss }}^{\xi}=\int_{t} \partial_{t} \rho_{\text {m,gliss }}^{\xi} d t$. As expected, the density reduction terms $\rho_{\text {gliss }}^{\xi}$ are always negative, whereas the generation terms $\rho_{\mathrm{m}, \mathrm{gliss}}^{\xi}$ are always positive. On the inactive slip

15 system 3, the dislocation density is generated by a deposition of dislocations as a consequence of the reaction between the two active slip systems 1 and 2 . The generation terms exceed the dislocation density reduction, since the inactive slip system only serves as a passive reaction partner. This leads to a net increase in dislocation density, which matches the increase observed on the inactive slip system as expected, cf. Fig. 6(a). In contrast, the density reduction terms

20 on the active slip systems 1 and 2 exceed the density generation, since the dislocation density on these slip systems is only generated by the reaction of one of the active slip systems with the inactive slip system 3, i.e. a passive reaction partner. Therefore, on the active slip systems, a net loss in dislocation density is observed due to the glissile reaction itself, as shown in Fig. 3. This can be understood by considering Eq. (13): one summand vanishes, if an inactive slip system

25 (no plastic slip rate) is involved. The slight decrease in dislocation density at the beginning of the simulation in Fig. 6(a) is a consequence of this behavior and the chosen initial conditions. 

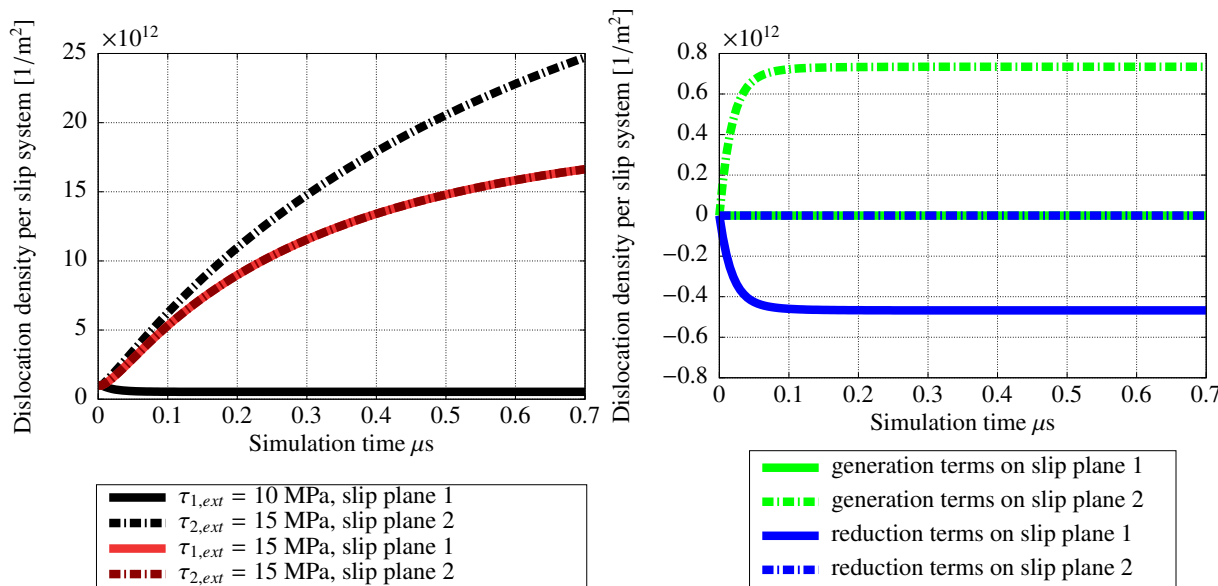

(a) Dislocation density per slip system with cross-slip. (b) Contribution of the cross-slip evolution equation for an unequal shear stress distribution.

Figure 7: Dislocation density per slip system over simulation time (a) and contribution of the cross-slip evolution equation by the density reduction and density generation terms integrated over the simulation time for an unequal shear stress distribution between both slip systems (b).

The dislocation density generation on active slip systems due to $\rho_{\mathrm{m}, \mathrm{gliss}}^{\xi}$ is always related to an increase in curvature density $\left(\partial_{t} q_{\mathrm{m}, \mathrm{gliss}}\right.$, Eq. (16)). The subsequent increase in total dislocation density, shown in Fig. 6(a), thus follows from the expansion of the newly generated dislocations due to the increase in curvature density.

\section{3.1.2. Cross-slip in a simplified system}

In a second analysis, we evaluate the cross-slip mechanism exclusively using the system setup described in section 2.3.1. Again, we use a local simulation, which neglects all density fluxes. First, the same initial dislocation density and the same shear stress of $\tau_{\text {ext }}=15 \mathrm{MPa}$ is chosen on both slip systems. Due to the assumption of symmetric cross-slip activation for equal shear 10 stress conditions, the contribution to dislocation density increase is also symmetric. Fig. 7(a) shows the results of the dislocation density evolution with a significant density increase, which evolves equally on both slip systems. In contrast to the glissile junction, the total dislocation density can not become less through cross-slip, since the lower bound of dislocation density evolution is equal dislocation density before and after the cross-slip.

In case of an unequal shear stress distribution between the slip systems, i.e. $\tau_{1, \text { ext }}=10 \mathrm{MPa}$ and $\tau_{2, \text { ext }}=15 \mathrm{MPa}$, the dislocation density on the slip system with higher stress increases, whereas the density on the slip system with lower shear stress slightly decreases. The contribution of the cross-slip evolution by the dislocation density reduction term $\rho_{\text {cross }}^{\xi}$ and the generation of new dislocation density $\rho_{\mathrm{m}, \text { cross }}^{\xi}$ according to Eq. (28) is shown in Fig. 7(b). The results show

20 that in case of unequal shear stress distribution, the cross-slip shifts the generation of dislocation density in direction of the slip system with higher shear stress.

\subsection{Dislocation multiplication in a fcc crystal with 12 slip systems}

For the comparison of the continuum formulation with the DDD results [1] we work with the [100] high-symmetry setup described in section 2.3.2. First, cross-slip is neglected and only 


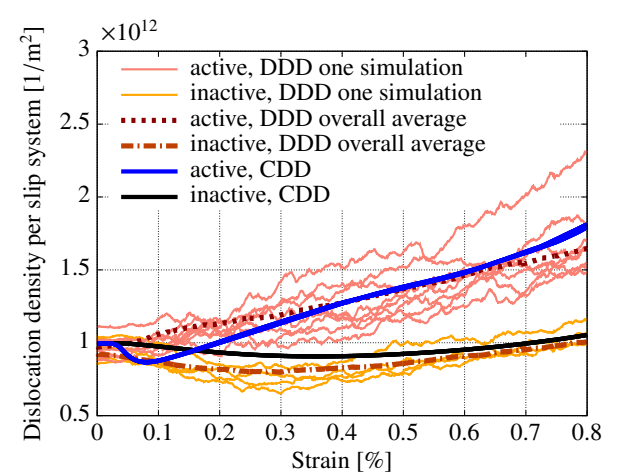

(a) Dislocation density per slip system.

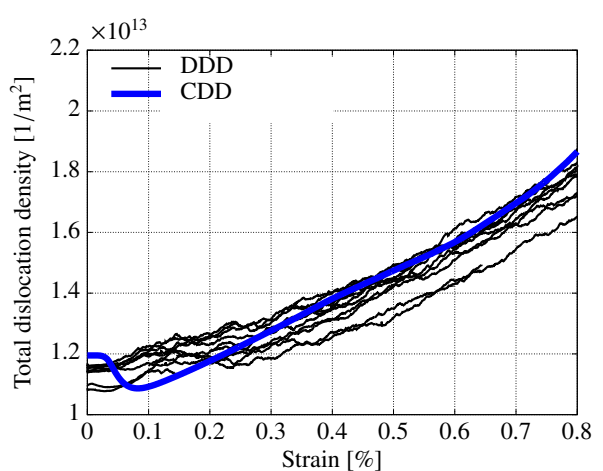

(b) Total dislocation density.

Figure 8: Dislocation density evolution per slip system with only glissile reactions compared to one DDD simulation and the average over 10 DDD simulations for active and inactive slip systems (a), total dislocation density compared to 10 DDD simulations (b) using the setup described in section 2.3.2. The CDD results in (a) are plotted for each slip system individually, in which the curves for active and inactive slip systems coincide, respectively.

glissile reactions contribute to dislocation multiplication as described in section 2.2.1. The results are shown in Fig. 8 in comparison with DDD data, which also were obtained considering the glissile mechanism only.

The evolution of the dislocation density is shown in Fig. 8(a) for the active as well as the 5 inactive slip systems. The results show that the glissile reactions lead to an increase of dislocation density on the active slip systems, whereas the reactions feeding into inactive slip systems merely deposit dislocation density with vanishing curvature. In contrast to CDD, the dislocation density evolution in DDD shows slight deviations between the different symmetric slip systems due to the statistical nature of the dislocation network. However, it can be seen that the CDD

10 dislocation density increase on both the active and inactive slip systems follows the respective DDD data on average. The dislocation density on the active slip systems initially declines, before increasing nearly linearly. Subsequently, the dislocation density on the inactive slip systems slightly increases with the increasing density on the active slip systems after about $0.4 \%$ strain.

To analyze the evolution of the total dislocation density in CDD, the evolution is compared

15 to 10 DDD simulations from [1] (Fig. 8(b)). The results show that the continuum model is able to reproduce the total density evolution observed in the DDD simulations quite accurately. The total dislocation density increase is about $50 \%$ for a strain of $0.8 \%$. However, an initial dip in the dislocation density evolution can be observed corresponding to the dips in the dislocation density evolution per slip system shown in Fig. 8(a).

Finally cross-slip and glissile reactions are combined for the simulation. In Fig. 9 the dislocation density evolution is depicted for the individual slip systems and for the total density, each time compared with according DDD results. The comparison to DDD shows that the evolution of dislocation density on both the active and inactive slip systems in CDD is in the same range for the given loading range (Fig. 9(a)). Particularly, the inactive slip systems show a significant 25 density increase in both the DDD and the continuum results. However, the dislocation density increase on active slip systems in DDD is almost linear, whereas the CDD results show a nonlinear behavior - CDD slightly underestimates the increase in dislocation density on the active slip systems in the beginning, while the density increase is overestimated later. Also for the inactive slip systems a larger dislocation density increase compared to DDD is observed after about $0.6 \%$ 


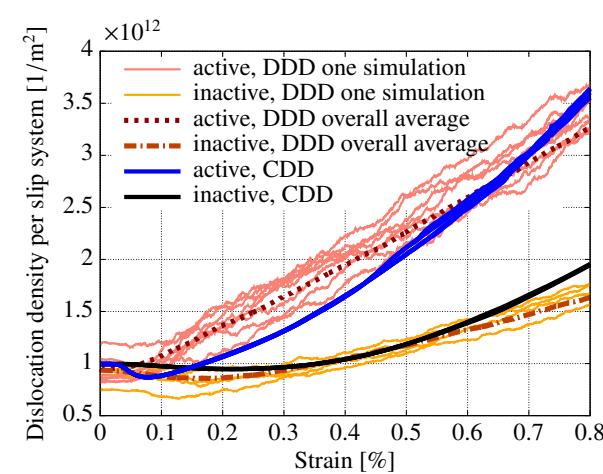

(a) Dislocation density per slip system.

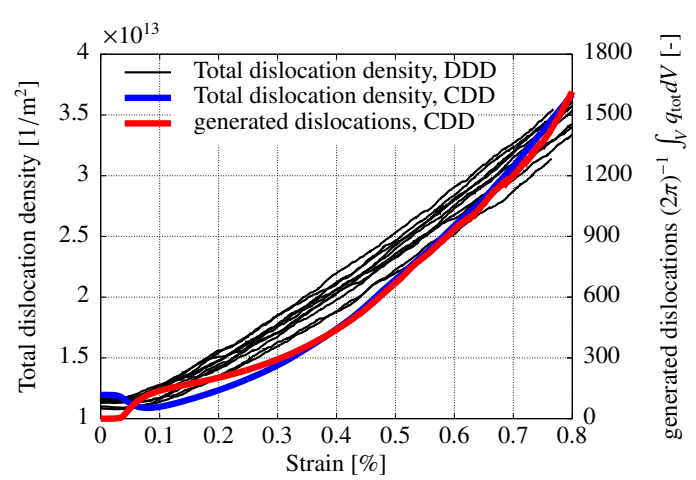

(b) Total dislocation density and number of generated dislocations.

Figure 9: Same as Fig. 8, but with glissile reactions and cross-slip. Additionally, the increase of the number of generated dislocations measured by the integral of the curvature density is shown in (b). The CDD results in (a) are plotted for each slip system individually.

strain.

Fig. 9(b) shows the total dislocation density as well as the evolution of the number of generated dislocations in comparison to 10 DDD simulations. This comparison serves to connect the increase in dislocation density to an increase in the number of dislocations in contrast to the 5 expansion of existing dislocations. It can be observed that the total dislocation density increases approximately by a factor of 3 until $0.8 \%$ strain and compares well to the corresponding DDD dislocation density evolution. Regarding the number of generated dislocations, the increase correlates with the increase in total dislocation density after $0.1 \%$ strain. Here, a factor of 10 can be measured, which is significantly more than the increase in dislocation density. However,

10 the dislocation density increase is clearly nonlinear with a small decrease after overcoming the yield stress and an overestimated density evolution compared to DDD in the second half of the simulation. The decline of dislocation density at the beginning (Fig. 9(a) and Fig. 9(b) and analogously in Fig. 8(a) and Fig. 8(b)) occurs due to the dislocation density flux through the outer surfaces, which dominates the total density evolution at this stage. This becomes particularly

15 obvious in the results in Appendix A. Here, the parameter choice $C_{3}=\pi / 4$ for Eq. (17) prevents a dislocation density reduction by the glissile reaction.

\section{Discussion}

A dislocation density based multiplication model including glissile and cross-slip mechanisms is introduced and compared to DDD data. In order to understand the evolution mecha-

20 nisms in the homogenized continuum approach, an isolated investigation of the mechanisms in simplified systems is conducted first and complemented by a tensile test of the full fcc system.

Analyzing the mechanisms in isolated systems show that includes glissile reactions leads to a net density increase on inactive slip systems. The density on inactive slip systems also acts as a passive partner for glissile reactions towards active slip systems, which leads to the generation of

25 new dislocation density and curvature density, i.e. a generation of a new dislocation. However, if the dislocation density and curvature density generation on active slip systems and the subsequent expansion of the newly generated dislocation is not able to overcome the loss of dislocation 
density due to the reaction with other slip systems, the total density decreases. This becomes particularly obvious in the beginning of the simulation in Fig. 6, where the initial condition of $q^{\xi}=0$ only allows for a density increase due to the glissile mechanism. In this case, the reaction serves as an annihilation term, which reduces the density until the generated curvature density 5 produces enough line length to overcome the density loss. Since the total dislocation density is contained in the dislocation density flux and contributes to the multiplication, there is no part of the density, which is stored in stable, or even immobile configurations. However, the results show that even in this simple setup the model is able to capture two key findings from DDD simulations: (1) multiplication mechanisms involve the generation of dislocation density as a 10 result of an interplay of different slip systems and (2) dislocation density is deposited on inactive slip systems.

Especially finding (2) is worthy to be analyzed more closely. The increasing density on the inactive slip system not only serves as a passive partner for further multiplication events, but also contributes to other dislocation reactions, whose effect on hardening is captured by the "Taylor"-

15 type flow-stress term. As a consequence, the inactive slip system is only "inactive" in the sense of a zero dislocation velocity. It does, however, contribute to hardening through an increase in dislocation density. This effect can be observed in the saturation of the density on the active slip systems (Fig. 6(a)) due to the flow-stress which is a function of the total dislocation density, i.e. including the increasing density on the inactive slip systems. It is obvious that a multiplication 20 model based on a slip-system-wise adoption of the Kocks-Mecking [7] theory would show no dislocation density evolution on an inactive slip system. As a consequence, an inactive slip system would not contribute to further dislocation interaction and hardening. The deposition of density on inactive systems is affecting cross hardening and possibly affects changing strain paths.

25 In contrast to the glissile reaction, cross-slip as a mechanism has no net loss in total dislocation density. Dislocation density is generated on the respective cross-slip system, where the bow-out of the dislocation takes effect, connected with a loss of dislocation density on the other slip system. Therefore the dislocation density increases significantly on the cross-slip systems. This is further amplified by the current assumption, that, as for glissile reactions, the cross-slip

so rate always involves a fraction of the total dislocation density if the conditions are met. As long as the stress is large enough and dislocation density exists, dislocation multiplication will take place. In case of an equal shear stress on two slip systems, the cross-slip probability is the same on both slip systems, see Fig. 7, thus we assume that cross-slip happens on both slip systems equally. This can be interpreted as double cross-slip processes, which are averaged into an

35 equal density increase on both slip systems connected with an expansion of the newly generated dislocations. In case of a different resolved shear stress on the primary and cross-slip system, multiplication takes place on the slip system with the higher resolved shear stress. The cross-slip mechanism of the our simple model is therefore close to the DDD cross-slip process and intended to reinforce the coupling of dislocation density by multiplication due to interplay of different slip

40 systems induced by the glissile reaction. However, for an averaging over many different (and possibly anisotropic) dislocation configurations, an analytic dislocation orientation distribution as e.g. proposed in [32] should be used.

Similar to the results in the simplified setup, the net dislocation density transfer between active slip systems in the full setup is zero. This leads to an equal dislocation density increase

45 on active and inactive slip systems, respectively. The dislocation density increase on the active and inactive slip systems as well as the increase in total dislocation density observed in DDD are captured well by the continuum model as shown in Fig. 8. However, the slight dislocation 
density decrease right after the yield point is observed even for an exclusion of the annihilation effect of the glissile reaction (Eqs. 15 and 17), as shown in the Appendix A, and can be related to the fact that a significant amount of line length leaves the simulation volume in the initial phase of the simulation. Due to the numerical inhibition of any dislocation motion on inactive

5 slip systems, where boundary conditions can affect the velocity field despite a vanishing nominal Schmid-factor, the dislocation density evolution on these systems can be observed as a result of the investigated mechanism, i.e. glissile reactions, only. Comparing the results in Fig. 8 to the simplified system in Fig. 6 yields the following conclusion for inactive slip systems: The combined effect of all 48 slip system pair combinations of the glissile reaction yields a dislocation density annihilation due to reactions of active with inactive slip systems, and a dislocation density increase due to deposition of reaction products. Here, the complex interplay of different densities and velocities on the slip systems, represented by the glissile evolution equation, leads to different glissile rates depending on whether or not an inactive slip system is involved in the reaction. The evolution of the dislocation density on inactive slip systems therefore depends on the interplay 15 between active slip systems, which is in accordance with observations in DDD.

The addition of dislocation multiplication via cross-slip in the full system yields a higher dislocation density increase on all slip systems, including the four inactive slip systems. This observation is in accordance with the behavior of the DDD-simulations (Fig. 9) and further corroborated by observations in [33] where it also is shown that cross-slip increases the number of junctions. It has to be remarked that the good agreement of the CDD results with DDD is achieved without introducing any additional fitting parameter compared to the simulations without cross-slip other than using the same cross-slip model as in DDD. Here, the characteristics of the presented model to couple the density increase on different slip systems with the considered multiplication mechanisms becomes particularly well visible: although there is no velocity on

25 the inactive slip systems, thus an influence of bow-out of the generated dislocations is excluded, a higher dislocation density increase is observed compared to the case without cross-slip (Fig. 8). This can be explained by a higher dislocation density production on active slip systems due to cross-slip, which yields again a higher rate of glissile reactions towards the inactive slip systems.

The integration of the curvature density on all slip systems can be interpreted as the number of closed dislocations, which are generated by the multiplication mechanisms. The observed correlation between the total dislocation density and the number of generated dislocations, as shown in Fig. 9(b), corresponds to observations in DDD. Furthermore, starting from about $0.1 \%$ strain until the end of the simulation, the number of generated dislocations increase by a factor of 10 , whereas the line length increases only by a factor of 3 . This indicates an increase of the 35 average dislocation curvature in the system over time, and thus forms the basis for the description of an increasingly dense dislocation network.

However, it can be observed that the rate of dislocation density increase is overestimated. This is due to the fact that by focusing on dislocation multiplication, further mechanisms which limit the dislocation density increase are not accounted for. In DDD the expansion of dislocations

40 is limited by their involvement in further reactions, where large parts of the angular change of the line orientation is equilibrated in end-nodes of dislocation junctions [1]. In the CDD framework, the consideration of dislocation annihilation, rather stable Lomer junctions and entangled dislocations could lead to a limitation of the dislocation density increase as observed from dislocations in networks.

Summarizing, the current work shows that the presented multiplication model is able to reproduce key findings from DDD simulations [1]: Dislocation multiplication is a result of mechanisms involving an interplay of dislocation density between slip systems and the dislocation 
density increase is largely driven by the generation of new dislocations instead of an expansion of existing dislocations. Furthermore, the glissile reaction plays an important role particularly for the dislocation density evolution on inactive slip systems. These findings are attained by the ability of the model to generate new dislocation density and curvature density based on the interplay

5 of dislocation densities and the coupling between slip systems. By generating curvature density along with dislocation density, closed dislocations are generated, which are able to produce line length by dislocation loop expansion. Compared to existing models, e.g. [2, 3, 4, 5, 6], which are driven by plastic slip on the same slip system, the multiplication process itself is independent of the plastic slip on the slip system of the new dislocation. Due to the resulting prevention of 10 self-replication of dislocation density, the presented model shows results per slip system closer to DDD observations than would be possible with existing derivates of the Kocks-Mecking-based multiplication model [7]. Thus, the proposed multiplication model provides a promising first approach to extend the available CDD formulation of planar expanding dislocation loops by including mechanisms of evolving dislocation networks. The model mimics the DDD observations

15 by including multiplication mechanisms, which involve an interplay of dislocations on different slip systems as induced by cross-slip and glissile reactions. The approach therefore provides a basis for the description of the evolution of dislocation networks, which is demonstrated using example systems.

However, there remain several open questions for the $\mathrm{CDD}$ formulation, providing a basis for future research. First, an improved cross-slip model could be formulated based on homogenizing further DDD cross-slip mechanisms such as e.g. proposed by [33]. Thereby, it could be accounted for further mechanisms in the continuum formulation, which are based on features of the dislocation microstructure instead of using a mechanism, which is solely driven by the resolved shear stress. Furthermore, by accounting for an analytic orientation distribution as proposed

25 in [32], the cross-slip model can potentially be generalized for use in arbitrary microstructure configurations. Second, since the curvature density in the present form does not allow to discriminate between curvature along the dislocation line and concentrated at single junction nodes, the representation of mechanisms leading to the formation of stable dislocation networks, e.g. by incorporating stable or sliding end-nodes of dislocation junctions is not yet possible. Therefore,

30 the contribution of line length production by loop expansion is also supposed to be overestimated in this case. To overcome this problem, the formulation should include "stored" dislocation curvature in stable or fixed end-nodes of dislocation junctions. Apart from glissile reactions, an improved formulation could also include Lomer reactions and annihilation mechanisms, like the collinear reaction, which limits the expansion of dislocations and provide a basis for the stability

35 of dislocation networks. Further, dislocation network mobility should incorporate a description, which captures the high scattering regarding the bow-out length and average travel distance of dislocations in the DDD-simulation [1]. Therefore, some of the phenomenological assumptions in the current model should be replaced by kinematically closed formulations for the evolution e.g. of dislocation segments instead of closed loops.

\section{Conclusion}

We introduce a dislocation multiplication model in the context of a dislocation based formulation of crystal plasticity, which addresses the challenge of a direct homogenization of discrete multiplication mechanisms through cross-slip and glissile reactions. The model is based on the generation of new dislocation density at the expense of dislocation density on one (in case of cross-slip) or two (in case of glissile reactions) other slip systems. Therefore, a key feature is 
that the multiplication formulation couples the dislocation density evolution between different slip systems.

The results show that the interplay of dislocation densities between the different slip systems leads to a density evolution on all slip systems akin to DDD-simulations. Particularly, the distri-

5 bution of dislocation density distinguished between active and inactive slip systems is reproduced correctly. The coupling of the dislocation density evolution on multiple slip systems correctly accounts for the deposition of dislocation density on inactive systems due to glissile reactions. In contrast to multiplication models which are based on line length increase related to plastic slip, dislocation density increase on inactive systems is able to contribute to further dislocation inter10 action. On active slip systems, new mobile dislocations are generated and successively expand due to the generation of curvature density contributing also to further plasticity and hardening. Additionally, the results show that the generation of new dislocations in the continuum formulation correlates well with the number of generated dislocation in DDD.

The work gives a first extension of the kinematic framework of CDD, which is based on the evolution of closed dislocation loops within their respective glide planes, towards including topological aspects of evolving dislocation networks. An open issue is e.g. the proper translation of dislocation curvature, which is equilibrated at junction nodes within the network and thus no longer available for dislocation line length production, to enable a meaningful representation of stable dislocation networks.

\section{Acknowledgements}

This work was performed on the computational resource ForHLR II funded by the Ministry of Science, Research and the Arts Baden-Wuerttemberg and DFG ('Deutsche Forschungsgemeinschaft"). The Financial support for the research group FOR1650 Dislocation based Plasticity funded by the German Research Foundation (DFG) under the contract numbers WE3544/5-2

25 (DW, MSt), GU367/36-2 (KS, MSu) and HO4227/5-1 (TH) as well as the support by the European Social Fund and the state of Baden-Wuerttemberg is gratefully acknowledged.

\section{Appendix A. Fcc crystal with different glissile reaction parameters}

In the current Appendix, we investigate the net density loss due to the choice of parameters in our model for the glissile reaction. The model as described by Eqs. (10) - (19) involves a dis-

30 location density decrease on the reacting slip systems (Eq. 17). If the parameters $C_{2}$ in Eq. (15) and $C_{3}$ in Eq. (17) are chosen as $C_{3}>\frac{\pi}{4} C_{2}$, the glissile process leads to a net density annihilation, which has to be overcome by expansion of the generated dislocations in order to result in an overall dislocation density increase. In contrast to the results presented before, here, the parameters are chosen as $C_{3}=\frac{\pi}{4} C_{2}$ with $C_{2}=1$. For this limiting case, the density reduction

35 and production cancels out, thus there is no overall change in line length for the glissile process depicted in Fig. 1 by combining Eqs. (15) and (17). The simulation setup is chosen as described in section 2.3.2 considering glissile reactions and cross-slip. The parameter $C_{1}$ in Eq. (13) is chosen as $C_{1}=0.032$ in order to scale the dislocation density production. This allows a dislocation density increase per slip system in the range of the DDD results, as shown in Fig. A.10. It is

40 observed, that the dislocation density on active slip systems initially slightly decreases similar to Fig. 9. Furthermore, the increase in dislocation density is overestimated on all slip systems after a strain of $0.4 \%$. 


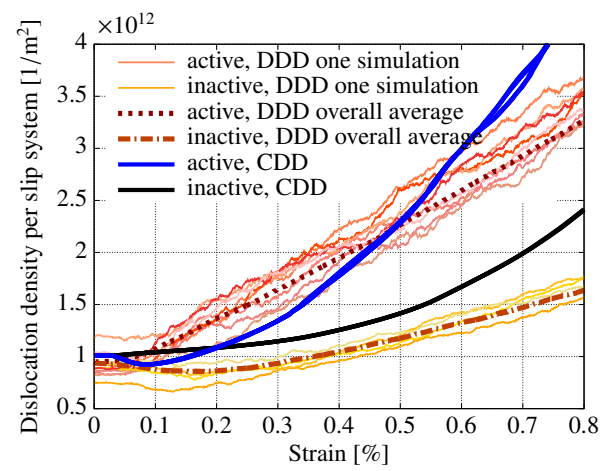

Figure A.10: Same as Fig. 9(a), but with $C_{1}=0.032$ and $C_{3}=\frac{\pi}{4}$. The CDD results are plotted for each slip system individually.

In order to have a detailed look at the contribution of the glissile reaction process, we consider the averaged dislocation density in the inner part of the system given by a cubic volume with half of the edge length of the full system. As explained in section 2.3.2, the initial dislocation density in this area is approximately homogeneous. By minimizing the impact of the system 5 boundary, we thus investigate the contribution of the multiplication mechanisms to the change of the dislocation density evolution. The comparison of the density evolution for the two parameter sets (according to Fig. A.10 and Fig. 9) up to $0.1 \%$ strain are given in table A.1. It can be seen that

\begin{tabular}{cccccc}
\hline \multirow{2}{*}{ Strain $[\%]$} & \multicolumn{2}{c}{$C_{1}=0.032, C_{3}=\pi / 4$} & & \multicolumn{2}{c}{$C_{1}=0.064, C_{3}=1$} \\
\cline { 2 - 3 } \cline { 5 - 6 } & $\rho\left[1 / \mu \mathrm{m}^{2}\right]$ active & $\rho\left[1 / \mu \mathrm{m}^{2}\right]$ inactive & & $\rho\left[1 / \mu \mathrm{m}^{2}\right]$ active & $\rho\left[1 / \mu \mathrm{m}^{2}\right]$ inactive \\
\hline 0 & 1.60 & 1.60 & 1.60 & 1.60 & 1.60 \\
0.02 & 1.60 & 1.61 & & 1.60 & 1.60 \\
0.04 & 1.60 & 1.63 & & 1.58 & 1.60 \\
0.06 & 1.65 & 1.66 & & 1.82 & 1.60 \\
0.08 & 1.80 & 1.69 & 2.04 & 1.61 \\
0.1 & 1.97 & & & 1.63 \\
\hline
\end{tabular}

Table A.1: Dislocation density evolution on active and inactive slip systems averaged over a central cubic volume with half of the edge length of the full system up to $0.1 \%$ strain. Comparison of two different parameter sets $C_{1}=0.032, C_{3}=$ $\frac{\pi}{4}$ (shown in Fig. A.10) and $C_{1}=0.064, C_{3}=1$ (shown in Fig. 9).

for the parameter set of Fig. A.10 the dislocation density is stable in the evaluated volume on both types of slip systems until a density increase is observed due to the multiplication mechanisms.

10 For the parameter set in Fig. 9, a minimal decrease in density prior to the increase is observed for the active systems only.

For further analysis of the density dip observed in Fig. A.10, the dislocation density leaving the outer surfaces of the simulation volume can be evaluated by measuring the dislocation flux on all slip systems through the outer system surfaces. The dislocation density leaving the system

15 is measured to be $0.22 \times 10^{12} 1 / \mathrm{m}^{2}$ until a strain of $0.1 \%$. This confirms, that the dips are caused by the boundary impact on the dislocation flux. 


\section{Appendix B. Perspectives for the cross-slip model}

For the cross slip model in Section 2 we assumed a distribution of the dislocation density on the circle of directions in the primary slip plane (indexed with $p$ ) based on the total dislocation density $\rho^{p}$ and the GND vector $\boldsymbol{\kappa}^{p}$. Before we discuss the assumed distribution underlying the 5 model in the main paper we present a general approach for obtaining the amount of dislocation density which can cross slip. Following [30] we assume that cross slip is possible for dislocations having a line direction within an angular interval $\pm \Delta \varphi$ around the positive and negative screw dislocation $\left(\Delta \varphi=10^{\circ}=\pi / 18\right.$ in the main paper).

We assume a distribution of density $\rho^{p}(\varphi)$ on the space of directions parametrized by the angle 10 $\varphi$ a dislocation line includes with the (positive) screw direction. With this convention positive and negative screw directions are found at $\varphi=0$ and $\varphi=\pi$, respectively. The distribution $\rho^{p}(\varphi)$ must be non-negative and has to fulfill the following constraints,

$$
\begin{aligned}
\int_{0}^{2 \pi} \rho^{p}(\varphi) \mathrm{d} \varphi & =\rho^{p}, \\
\int_{0}^{2 \pi} \rho^{p}(\varphi)(\cos \varphi, \sin \varphi) \mathrm{d} \varphi & =\left(\kappa_{\mathrm{s}}^{p}, \kappa_{\mathrm{e}}^{p}\right),
\end{aligned}
$$

where $\kappa_{\mathrm{s}}^{p}$ and $\kappa_{\mathrm{e}}^{p}$ denote the screw component and the edge component of the GND vector $\boldsymbol{\kappa}^{p}$.

Given such a distribution function, the total amount of density which might cross slip is obtained as

$$
\tilde{\rho}^{p}=\int_{-\Delta \varphi}^{\Delta \varphi} \rho^{p}(\varphi) \mathrm{d} \varphi+\int_{\pi-\Delta \varphi}^{\pi+\Delta \varphi} \rho^{p}(\varphi) \mathrm{d} \varphi .
$$

Any reasonable assumption on $\rho^{p}(\varphi)$ therefore yields a possible cross slip density $\tilde{\rho}^{p}$ needed for the cross slip model introduced in the main text.

The assumption underlying the cross slip model in the current work is

$$
\rho^{p}(\varphi)=\frac{1}{2 \pi}\left(\rho^{p}-\left\|\kappa^{p}\right\|\right)+\left\|\kappa^{p}\right\| \delta_{\varphi_{\kappa}},
$$

where $\varphi_{K}$ denotes the angle which the GND direction includes with the screw direction, and $\delta_{\varphi_{k}}$ denotes the Dirac delta-distribution located at this angle. Inserted into (B.3), the constant part yields what is called SSD part in the main text and the delta distribution yields the Heaviside function term, as the integrals over the delta distribution evaluates to 1 if the angle $\varphi_{\kappa}$ is within the integration interval and to 0 else. The Heaviside function is the reason that in this model the fraction of possible cross slip density may change discontinuously for high GND contents with a direction close to the border of the cross slip regions defined by the angle $\Delta \varphi$.

Smooth options for assuming the density $\rho^{p}(\varphi)$ are available in the literature. A simple ansatz is to use a truncated tensor expansion [12] (equivalent to a truncated Fourier expansion [11]), such that

$$
\rho^{p}(\varphi)=\frac{1}{2 \pi}\left[\rho^{p}+2 \boldsymbol{\kappa} \cdot \boldsymbol{l}(\varphi)+4 \boldsymbol{l}(\varphi) \cdot \boldsymbol{A}^{p} \cdot \boldsymbol{l}(\varphi)\right],
$$

with $\boldsymbol{A}^{p}$ obtained from the closure assumption (4) and $\boldsymbol{l}(\varphi)=(\cos \varphi, \sin \varphi)$.

The presumably best ansatz for assuming the density $\rho^{p}(\varphi)$ derives from a maximum information entropy principle $[32,34]$. This yields the density as a multiple of a von Mises distribution around the GND direction in the form

$$
\rho^{p}(\varphi)=\frac{\rho^{p}}{2 \pi I_{0}(\Lambda)} \exp _{25}\left(\Lambda \cos \left(\varphi-\varphi_{\kappa}\right)\right),
$$


where $\Lambda$ is obtained from

$$
\frac{I_{1}(\Lambda)}{I_{0}(\Lambda)}=\frac{\left\|\boldsymbol{\kappa}^{p}\right\|}{\rho^{p}}
$$

with $I_{0}$ and $I_{1}$ denoting modified Bessel functions of first kind. In order to use this maximum entropy ansatz the latter equation needs to be (approximately) solved for $\Lambda$ and an approximation for the cumulative distribution function of the von Mises distribution would be required to

5 simplify evaluating the integrals in (B.3).

\section{References}

[1] M. Stricker, M. Sudmanns, K. Schulz, T. Hochrainer, and D. Weygand. Dislocation multiplication in stage ii deformation of fcc multi-slip single crystals. Journal of the Mechanics and Physics of Solids, 2018.

[2] A. Ma, F. Roters, and D. Raabe. A dislocation density based constitutive model for crystal plasticity FEM including geometrically necessary dislocations. Acta Materialia, 54(8):2169-2179, 2006.

[3] L. Kubin, B. Devincre, and T. Hoc. Modeling dislocation storage rates and mean free paths in face-centered cubic crystals. 56(20):6040 - 6049.

[4] L. Kubin, B. Devincre, and T. Hoc. Toward a physical model for strain hardening in fcc crystals. 483:19-24.

[5] C. Reuber, P. Eisenlohr, F. Roters, and D. Raabe. Dislocation density distribution around an indent in singlecrystalline nickel: Comparing nonlocal crystal plasticity finite-element predictions with experiments. Acta materialia, 71:333-348, 2014.

[6] F Roters, M Diehl, P Shanthraj, P Eisenlohr, C Reuber, SL Wong, T Maiti, A Ebrahimi, T Hochrainer, HO Fabritius, et al. Damask - the duesseldorf advanced material simulation kit for modelling multi-physics crystal plasticity, damage, and thermal phenomena from the single crystal up to the component scale. Comput. Mater. Sci, 2018.

[7] U. F. Kocks and H. Mecking. Physics and phenomenology of strain hardening: The fcc case. Progress in materials science, 48(3):171-273, 2003.

[8] A. Arsenlis and D. M. Parks. Modeling the evolution of crystallographic dislocation density in crystal plasticity. Journal of the Mechanics and Physics of Solids, 50(9):1979-2009, 2002.

[9] A. Alankar, D. P. Field, and H. M. Zbib. Explicit incorporation of cross-slip in a dislocation density-based crystal plasticity model. Philosophical Magazine, 92(24):3084-3100, 2012.

[10] H.S. Leung, P.S.S. Leung, B. Cheng, and A.H.W. Ngan. A new dislocation-density-function dynamics scheme for computational crystal plasticity by explicit consideration of dislocation elastic interactions. International Journal of Plasticity, 67:1-25, 2015.

[11] T. Hochrainer, S. Sandfeld, M. Zaiser, and P. Gumbsch. Continuum dislocation dynamics: Towards a physical theory of crystal plasticity. Journal of the Mechanics and Physics of Solids, 63:167 - 178, 2014.

[12] T. Hochrainer. Multipole expansion of continuum dislocations dynamics in terms of alignment tensors. Philosophical Magazine, 95(12):1321-1367, 2015.

[13] T. Hochrainer. Thermodynamically consistent continuum dislocation dynamics. Journal of the Mechanics and Physics of Solids, 88:12-22, 2016.

35 [14] S. Sandfeld and G. Po. Microstructural comparison of the kinematics of discrete and continuum dislocations models. 23(8):085003.

[15] K. Schulz, D. Dickel, S. Schmitt, S. Sandfeld, D. Weygand, and P. Gumbsch. Analysis of dislocation pile-ups using a dislocation-based continuum theory. Modelling and Simulation in Materials Science and Engineering, 22(2):025008, 2014

40 [16] S. Schmitt, P. Gumbsch, and K. Schulz. Internal stresses in a homogenized representation of dislocation microstructures. Journal of the Mechanics and Physics of Solids, 84:528-544, 2015.

[17] K. Schulz, M. Sudmanns, and P. Gumbsch. Dislocation-density based description of the deformation of a composite material. Modelling and Simulation in Materials Science and Engineering, 25(6):064003, 2017.

[18] M. Sudmanns, P. Gumbsch, and K. Schulz. Plastic flow and dislocation strengthening in a dislocation density based formulation of plasticity. Computational Materials Science, 151:317-327, 2018.

[19] S. Sandfeld and T. Hochrainer. Towards frank-read sources in the continuum dislocation dynamics theory. AIP Conference Proceedings, 1389(1):1531-1534, 2011.

[20] Y. Zhu, H. Wang, X. Zhu, and Y. Xiang. A continuum model for dislocation dynamics incorporating frank-read sources and hallpetch relation in two dimensions. International Journal of Plasticity, 60:19-39, 2014

50 [21] S. Schmitt, M. Stricker, P. Gumbsch, and K. Schulz. A mechanism-based homogenization of a dislocation source model for bending. Acta Materialia, 2018. 
[22] M. Monavari and M. Zaiser. Annihilation and sources in continuum dislocation dynamics. Materials Theory, 2(1), 2018.

[23] M. Stricker and D. Weygand. Dislocation multiplication mechanisms - glissile junctions and their role on the plastic deformation at the microscale. Acta Materialia, 99:130-139, 2015.

5 [24] G. I. Taylor. The mechanism of plastic deformation of crystals. part i. theoretical. Proceedings of the Royal Society of London. Series A, Containing Papers of a Mathematical and Physical Character, 145(855):362-387, 1934

[25] K. Schulz, L. Wagner, and C. Wieners. A mesoscale approach for dislocation density motion using a runge-kutta discontinuous galerkin method. PAMM, 16(1):403-404, 2016.

[26] C. Wieners. A geometric data structure for parallel finite elements and the application to multigrid methods with block smoothing. Computing and Visualization in Science, 13(4):161-175, 2010.

[27] C. Wieners. Distributed point objects. a new concept for parallel finite elements. In Timothy J. Barth, Michael Griebel, David E. Keyes, Risto M. Nieminen, Dirk Roose, Tamar Schlick, Ralf Kornhuber, Ronald Hoppe, Jacques Priaux, Olivier Pironneau, Olof Widlund, and Jinchao Xu, editors, Domain Decomposition Methods in Science and Engineering, volume 40, pages 175-182. Springer-Verlag, 2005.

15 [28] L. P. Kubin. Dislocation microstructures and plastic flow: a 3d simulation. Solid State Phenomena, 1992.

[29] M. Verdier, M. Fivel, and I. Groma. Mesoscopic scale simulation of dislocation dynamics in fcc metals: Principles and applications. Modelling and Simulation in Materials Science and Engineering, 6(6):755, 1998.

[30] D. Weygand, L. H. Friedman, E. van der Giessen, and A. Needleman. Aspects of boundary-value problem solutions with three-dimensional dislocation dynamics. Modelling and Simulation in Materials Science and Engineering, 10(4):437, 2002

[31] K. Schulz, L. Wagner, and C. Wieners. A mesoscale continuum approach of dislocation dynamics and the approximation by a runge-kutta discontinuous galerkin method. International Journal of Plasticity, 120:248 - 261, 2019.

[32] Mehran Monavari, Michael Zaiser, and Stefan Sandfeld. Comparison of closure approximations for continuous dislocation dynamics. MRS Online Proceedings Library Archive, 1651, 2014.

[33] A. M. Hussein, S. I. Rao, M. D. Uchic, D. M. Dimiduk, and J. A. El-Awady. Microstructurally based cross-slip mechanisms and their effects on dislocation microstructure evolution in fcc crystals. Acta Materialia, 85:180-190, 2015.

[34] M. Monavari, S. Sandfeld, and M. Zaiser. Continuum representation of systems of dislocation lines: A general method for deriving closed-form evolution equations. Journal of the Mechanics and Physics of Solids, 95:575601, October 2016 